\title{
Simultaneous skin friction and velocity measurements in high Reynolds number pipe and boundary layer flows
}

\author{
R. Baidya ${ }^{1} \dagger$, W. J. Baars ${ }^{1}$, S. Zimmerman ${ }^{1}$, M. Samie ${ }^{1}$, R. J. Hearst ${ }^{2,3}$, E. Dogan ${ }^{2}$, \\ L. Mascotelli ${ }^{4}$, X. Zheng ${ }^{4}$, G. Bellani ${ }^{4}$, A. Talamelli $^{4}$, B. Ganapathisubramani $^{2}$, N. \\ Hutchins $^{1}$, I. Marusic ${ }^{1}$, J. Klewicki ${ }^{1}$ and J. P. Monty ${ }^{1}$ \\ ${ }^{1}$ Department of Mechanical Engineering, University of Melbourne, Melbourne, Victoria 3010, Australia \\ ${ }^{2}$ Aerodynamics and Flight Mechanics Research Group, University of Southampton, Hampshire, SO17 \\ 1BJ, UK \\ ${ }^{3}$ Department of Energy \& Process Engineering, Norwegian University of Science \& Technology, \\ Trondheim, NO-7491, Norway \\ ${ }^{4}$ Department of Industrial Engineering, CIRI Aerospace, Alma Mater Studiorum, Università di Bologna, \\ 47100 Forlì, Italy
}

(Received $\mathrm{xx}$; revised $\mathrm{xx}$; accepted $\mathrm{xx}$ )

Streamwise velocity and wall-shear stress are acquired simultaneously with a hot-wire and an array of azimuthal/spanwise-spaced skin friction sensors in large-scale pipe and boundary layer flow facilities at high Reynolds numbers. These allow for a correlation analysis on a per-scale basis between the velocity and reference skin friction signals to reveal which velocity-based turbulent motions are stochastically coherent with turbulent skin friction. In the logarithmic region, the wall-attached structures in both the pipe and boundary layers show evidence of selfsimilarity, and the range of scales over which the self-similarity is observed decreases with an increasing azimuthal/spanwise offset between the velocity and the reference skin friction signals. The present empirical observations support the existence of a self-similar range of wall-attached turbulence, which in turn are used to extend the model of Baars et al. (J. Fluid Mech., vol. 823, 2017, R2) to include the azimuthal/spanwise trends. Furthermore, the region where the self-similarity is observed correspond with the wall height where the mean momentum equation formally admits a self-similar invariant form, and simultaneously where the mean and variance profiles of the streamwise velocity exhibit logarithmic dependence. The experimental observations suggest that the self-similar wall-attached structures follow an aspect ratio of $7: 1: 1$ in the streamwise, spanwise and wall-normal directions, respectively.

Key words:

\section{Introduction}

Following the discovery of quasi-periodic features within wall-bounded turbulence that are thought to be associated with the physical mechanism that governs turbulence - from production at the expense of the mean flow to eventual dissipation due to viscous forces at the fine scales (Robinson 1991), substantial efforts have been made to better understand these critical processes (Jiménez 2011). The quasi-periodic features remain coherent across a finite three-dimensional

† Email address for correspondence: baidyar@unimelb.edu.au 
domain, and in this study we focus on the coherent structures that reside in the inertial range of the energetic scales that become increasingly prominent at high Reynolds number, and therefore account for a large portion of overall turbulence production (Smits et al. 2011). One of the challenges of examining high Reynolds number flows is to capture the broadband turbulence over the extensive range of scales, which by definition of Reynolds number $(R e)$ corresponds to the separation of scales between the smallest and the largest energetic motions present within a flow. From an experimental point of view, the range of scales accessible is typically constrained by the physical size of the sensor at the small scales and the facility for the large scales. Hence, our approach here is to utilise large-scale facilities, that allow high $R e$ flows while still maintaining the smallest energetic length scales such that they are accessible using conventional techniques. Here, we present novel pipe flow experiments where an azimuthal array of skin friction signals are simultaneously sampled with a velocity sensor. Due to the simplicity of axially symmetric mean flow, the fully developed pipe flow is a classical configuration to examine wall-bounded turbulence. The results from the pipe flow are also compared against a turbulent boundary layer dataset, where simultaneous skin friction measurements with velocity have been acquired.

One of the conceptual models for wall-bounded flows which has received considerable attention is the attached eddy hypothesis (AEH) proposed by Townsend (1976). The AEH idealises wall-bounded flow as a collection of inertia-driven coherent structures that are self-similar and are randomly distributed in the plane of the wall. The AEH prescribes these coherent structures, or eddies, to scale with the distance from the wall with the height of the eddies following a geometric progression (Perry \& Chong 1982). To assess the self-similarity of coherent structures in a wall-bounded flow, del Álamo et al. (2006) examined their size based on dimensions of a vortex core identified by thresholding the discriminant of the velocity gradient tensor, using direct numerical simulation (DNS) datasets. They find that the tall vortex clusters which extend from the near wall (below 20 viscous units) to the logarithmic region, scale with wall height. Furthermore, work by Hwang (2015) suggests that these self-similar structures can self-sustain and therefore play a key role in driving the wall-bounded turbulence. Experimentally, fully resolving the velocity gradient tensor is challenging, and typically the streamwise velocity is used as a surrogate. For example, Hellström et al. (2016) uses Proper Orthogonal Decomposition (POD) on instantaneous snapshots of the streamwise velocity from a radial-azimuthal plane in a pipe flow. They find that the POD mode shapes of the radial-azimuthal structure within the pipe flow follow a self-similar progression that obeys wall scaling. That is, the various POD mode shapes show a one-to-one relationship between azimuthal mode number and their characteristic wall-normal extent. An excellent overview of key assumptions and limitations associated with AEH is provided by Marusic \& Monty (2019).

When examining flow data in the context of the AEH, a common objective is to search for scaling laws in energy spectra (Baidya et al. 2017; Nickels et al. 2005) and wall-normal profiles of the turbulent stresses (Marusic et al. 2013). In energy spectra of the streamwise velocity component, a $k_{x}^{-1}$ behaviour in the inertial range (where $k_{x}$ corresponds to the streamwise wavenumber) would reflect a self-similar wall-attached structure of the turbulence envisioned in Townsend's AEH. Likewise, a semi-logarithmic wall-normal decay in the variance of the streamwise velocity also reflects such a structure. For several decades it has been challenging to observe these scaling laws in raw velocity data and conclusive empirical evidence has remained elusive (Marusic et al. 2010). Davidson \& Krogstad (2008) propose that the one-dimensional spectrum is not an ideal tool to investigate the self-similar behaviour due to an aliasing effect, whereby a large wavenumber, whose wavenumber vector is inclined with respect to the direction of measurement, is interpreted as a contribution to the one-dimensional $u$-spectra at a lower wavenumber (Tennekes \& Lumley 1972), presumably contaminating the $k_{x}^{-1}$ behaviour. Indeed, a clear trend towards self-similarity is evident in two-dimensional $u$-spectra (here the spectral energetic content can be examined as a function of streamwise and spanwise wavenumbers) with 
increasing $R e$, although this behaviour does not necessarily translate to a more prominent $k_{x}^{-1}$ behaviour once an integration is performed along the spanwise wavenumbers to obtain the onedimensional spectra (Chandran et al. 2017). Instead of $k_{x}^{-1}$ behaviour in the one-dimensional $u$-spectra, Davidson \& Krogstad (2008) advocate $\overline{(\Delta u)^{2}} \sim \log \left(r_{x}\right)$ scaling for the structure function as an indicator of self-similarity, where $\Delta u=u\left(x+r_{x}\right)-u(x)$ is the difference in the streamwise fluctuating velocity, $u$, separated in the streamwise direction, $x$, with a displacement $r_{x}$. Subsequent assessment performed by de Silva et al. (2015) showed a decade of $\log \left(r_{x}\right)$ behaviour in the structure function for high $R e$ boundary layer flow. Noticeable deviations from the logarithmic behaviour occur however for the pipe flow at a comparable Re (Chung et al. 2015). Chung et al. (2015) suggest that these differences in the $\overline{(\Delta u)^{2}}$ behaviour between the pipe and boundary layer flows are due to a crowding effect in the pipe, whereby a restriction is imposed on the width of the structures by its geometry. Furthermore, at an even higher $R e$ $\left(\operatorname{Re}_{\tau} \sim 10^{6}\right)$ universality of $\overline{(\Delta u)^{2}} \sim \log \left(r_{x}\right)$ behaviour is retained between the pipe and boundary layer flows. More recently, Yang et al. (2017) generalise the scaling of structure functions in arbitrary directions in the three-dimensional space based on the AEH.

In this paper, we will follow the approach of using a correlation-based metric to examine the wall-attached structure of the wall-bounded turbulence. As opposed to an assessment of $\overline{(\Delta u)^{2}} \sim \log \left(r_{x}\right)$ behaviour, which is based on correlation statistics at a single wall height practically computed by assuming Taylor's hypothesis, we here employ synchronised measures of turbulence at two wall-normal positions. A reference turbulent skin friction at the wall is acquired using hot-film sensors glued to the wall. These measurements are complemented by a sequence of velocity measurements performed at various wall-height $(z)$ locations. Using the two-point measurements, it can be revealed to what degree the turbulent scales in the flow remain coherent with the wall-reference signal. The aim of this paper is to characterise the coherent part of the velocity signal, associated with the wall-attached structures (Baars et al. 2016), as a function of its wavelength $\left(\lambda_{x}=2 \pi / k_{x}\right)$, transverse offset $(\Delta s)$ and wall-normal offset $(z)$; and to extend the observations by Baars et al. (2017) in the $\lambda_{x}-z$ plane to incorporate the $\Delta s$ trends.

\section{Experimental set-up}

Tables 1 and 2 list the experimental conditions and sensors utilised for the pipe and boundary layer datasets considered in this paper, while details of each experiment are given in subsequent paragraphs. Here, we report the friction Reynolds number, $R e_{\tau}=L_{O} U_{\tau} / v$, where $L_{O}, U_{\tau}$ and $v$ correspond to the outer length scale (pipe radius and boundary layer thickness), mean friction velocity and kinematic viscosity, respectively. While the pipe radius, $R$, can be directly measured; the boundary layer thickness, $\delta$, is determined here by fitting the mean velocity profile to a modified Coles law of the wall/wake formulation (see Perry et al. 2002). In addition, $x, y$ and $z$ denote the streamwise, spanwise and wall-normal directions, respectively; and superscript ' + ' indicates normalisation by viscous units (e.g. $U^{+}=U / U_{\tau}, z^{+}=z U_{\tau} / v$ and $\Delta t^{+}=\Delta t U_{\tau}^{2} / v$ ). Capitalisation and overbar denote time-averaged quantities, while lower cases correspond to fluctuations from the time-averaged mean values.

\subsection{Pipe flow}

The pipe flow experiments are conducted at the Centre for International Cooperation in Long Pipe Experiments (CICLoPE) facility belonging to the University of Bologna, located in Predappio, Italy. The inner diameter of this unique large-scale facility is $0.9 \mathrm{~m}$, with the measurement station located at the downstream end of a $111 \mathrm{~m}$ long pipe, where a fully developed turbulent pipe flow, for the first- and second-order statistics, is established (Örlü et al. 2017). The large dimension for the outer length scale, $R$, corresponding in this case to the pipe radius, 


\begin{tabular}{|c|c|c|c|c|c|c|c|c|}
\hline Flow type & $R e_{\tau}$ & Symbol & $\begin{array}{c}U_{C L}, U_{\infty} \\
\left(\mathrm{ms}^{-1}\right)\end{array}$ & $\begin{array}{l}R, \delta \\
(\mathrm{m})\end{array}$ & $\begin{array}{c}U_{\tau} \\
\left(\mathrm{ms}^{-1}\right)\end{array}$ & $\frac{v}{U_{\tau}}$ & $\begin{array}{l}T_{s} \\
(\mathrm{~s})\end{array}$ & $\frac{T_{s} U_{C L}}{R}, \frac{T_{s} U_{\infty}}{\delta}$ \\
\hline \multirow{3}{*}{ Pipe } & 10000 & $\Delta$ & 9.8 & \multirow{3}{*}{0.45} & 0.34 & 46 & 115 & 2500 \\
\hline & 21500 & a & 22.9 & & 0.74 & 21 & 100 & 5000 \\
\hline & 39500 & $\bullet$ & 43.5 & & 1.35 & 11 & 60 & 5800 \\
\hline $\begin{array}{c}\text { Boundary } \\
\text { layer }\end{array}$ & 14000 & $\diamond$ & 20.3 & 0.33 & 0.67 & 23 & 300 & 19000 \\
\hline
\end{tabular}

Table 1: Summary of experimental conditions. $U_{C L}$ and $U_{\infty}$ denote the centreline and free-stream velocities in the pipe and boundary layer flows, while $T_{s}$ correspond to the total sampling time at each wall-normal location, $z$.

\begin{tabular}{|c|c|c|c|c|c|c|c|c|c|c|c|c|}
\hline \multirow[b]{2}{*}{ Flow type } & \multirow[b]{2}{*}{$R e_{\tau}$} & \multicolumn{6}{|c|}{ Hot-wire details } & \multicolumn{5}{|c|}{ Hot-film details } \\
\hline & & $\begin{array}{c}d \\
(\mu \mathrm{m})\end{array}$ & $\begin{array}{c}l \\
(\mathrm{~mm})\end{array}$ & $l^{+}$ & $\overline{\mathrm{OHR}}$ & $\begin{array}{l}1 / \Delta t \\
(\mathrm{~Hz})\end{array}$ & $\overline{\Delta t^{+}}$ & $\begin{array}{c}l \\
(\mathrm{~mm})\end{array}$ & $l^{+}$ & $\overline{\mathrm{OHR}}$ & $\begin{array}{l}1 / \Delta t \\
(\mathrm{~Hz})\end{array}$ & $\overline{\Delta t^{+}}$ \\
\hline Pipe & $\begin{array}{l}10000 \\
21500 \\
39500\end{array}$ & 2.5 & 0.5 & $\begin{array}{l}11 \\
24 \\
44\end{array}$ & 1.8 & 65000 & $\begin{array}{l}0.12 \\
0.59 \\
1.97\end{array}$ & 1.5 & $\begin{array}{c}33 \\
72 \\
131\end{array}$ & 1.05 & 6500 & $\begin{array}{c}1.2 \\
5.9 \\
19.7\end{array}$ \\
\hline $\begin{array}{l}\text { Boundary } \\
\text { layer }\end{array}$ & 14000 & 2.5 & 0.5 & 21 & 1.8 & 60000 & 0.47 & 0.9 & 38 & 1.05 & 60000 & 0.47 \\
\hline
\end{tabular}

Table 2: Summary of velocity and skin friction sensors utilised. OHR denote the overheat ratio used for each sensor, while $1 / \Delta t$ corresponds to the sampling frequency.

allows access to high Reynolds numbers while the smallest energetic scales still remain $O(10 \mu \mathrm{m})$ and therefore can be resolved using conventional techniques (Talamelli et al. 2009). For further technical and flow characterisation details on the facility, the readers are referred to Bellani \& Talamelli (2016), Willert et al. (2017) and Örlü et al. (2017). The current experimental set-up consists of an array of 51 skin friction sensors located at various azimuthal positions spanning from $0-2 \pi$ along the pipe circumference simultaneously sampled with a hot-wire probe, nominally located at the same $x$ location as the hot-films. In addition, the hot-wire probe can be traversed from near the wall to the pipe centreline as shown in figure 1(a). It should be noted that the large pipe also allows the skin friction to be better resolved in the azimuthal direction by virtue of being able to accommodate a larger number of sensors along the circumference (see figure 1), compared to an alternate approach to high Reynolds numbers that relies on small $v / U_{\tau}$, typically achieved through reduction of the kinematic viscosity (e.g. Zagarola \& Smits 1998).

The velocity sensor is a hot-wire consisting of a Wollaston wire mounted onto a modified 55P15-type Dantec probe and etched to expose a $2.5 \mu \mathrm{m}$ diameter platinum core of $0.5 \mathrm{~mm}$ length. The platinum wire is heated and maintained at a constant temperature with an overheat ratio of 1.8 using a Dantec StreamLine Pro anemometer system. Furthermore, here we maintain a hot-wire length $(l)$ to diameter $(d)$ ratio of 200 to avoid contamination from end conduction (Ligrani \& Bradshaw 1987). Before and after each experiment, the hot-wire is traversed close to the centreline ( $\sim 0.93 R$ due to the limited range of the traverse) and the mean voltage is calibrated in situ against the centreline velocity, which is measured using a Pitot-static tube. This allows construction of a one-to-one relationship between the flow velocity and the anemometer output 

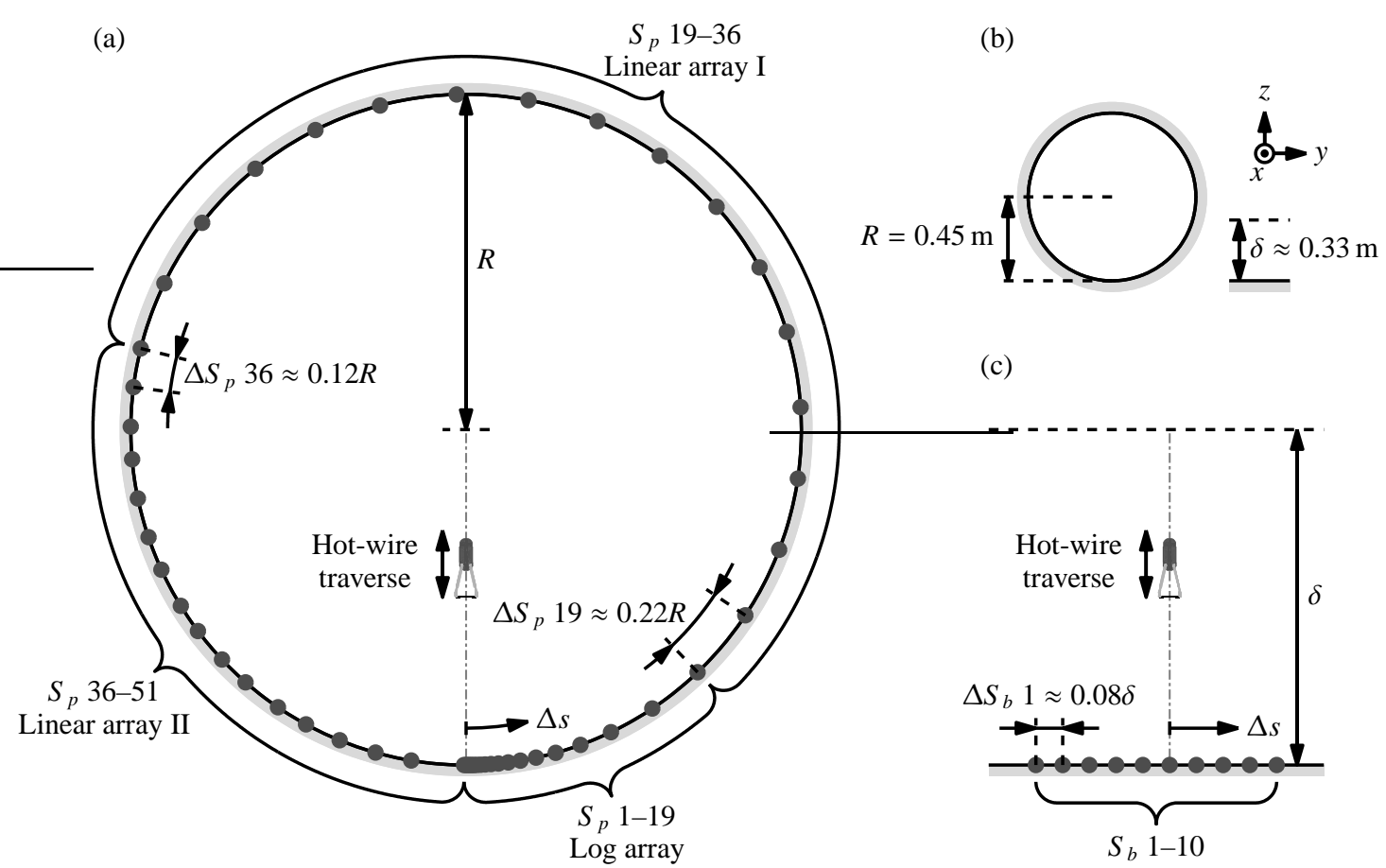

(c)

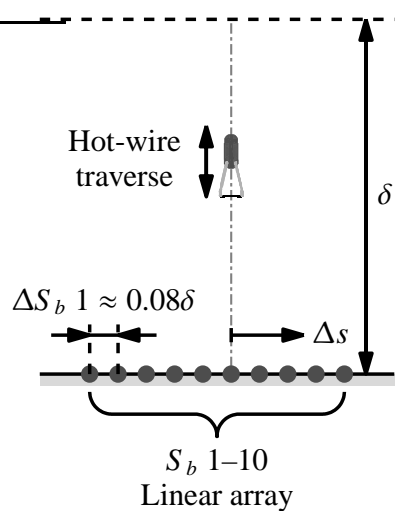

Figure 1: Schematic of experimental set-ups. (a,c) Show locations of hot-film (๑) and hot-wire sensors (not to scale) for the pipe and boundary layer flows, while (b) shows comparison of the outer length-scales $R$ and $\delta$ respectively, in physical units.

voltage. Although this means that calibration is not performed in a near-potential flow, the ratio $\sqrt{\overline{u^{2}}} / U$ at the centreline is sufficiently small to make insignificant differences to the potential flow calibration (Monty 2005; Örlü et al. 2017). An intermediate calibration relationship between the hot-wire voltages and velocities is generated for each wall-normal point during the measurement, based on an assumption that deviations between pre- and post-calibration curves are the result of a linear drift in the hot-wire voltage with time. Figure 2(a,b) shows comparisons of the streamwise velocity mean profiles from the pipe experiments (denoted by symbols) against the dataset of Ahn et al. (2015). Despite the departure from ideal calibration conditions, the $U$ profiles from the current datasets show good agreement with the DNS of Ahn et al. (2015) $(--)$ in the inner region when scaled in viscous units. In addition, a good agreement with the DNS is also observed for the deficit profiles in the outer region. Unlike the mean, the measured variance profiles are dependent on the sensor spatial resolution (Hutchins et al. 2009; Ligrani \& Bradshaw 1987). For the variance profiles shown in figures 2(c) and (d), the spatial resolution in the azimuthal direction varies from 6 viscous units between the adjacent grids (at the wall) for the DNS to 40 viscous units for the $R e_{\tau} \approx 40000$ dataset from the current experiment. However, since the influence of the spatial resolution diminishes with an increasing $z$, a good collapse of the $\overline{u^{2}}$ profiles is observed for the region $z / R>0.1$ in the outer scaling. Our $U$ and $\overline{u^{2}}$ results and the conclusions drawn agree with the findings of Örlü et al. (2017) from the same facility. Note, the present analysis is particularly insensitive to slight calibration differences (including the 

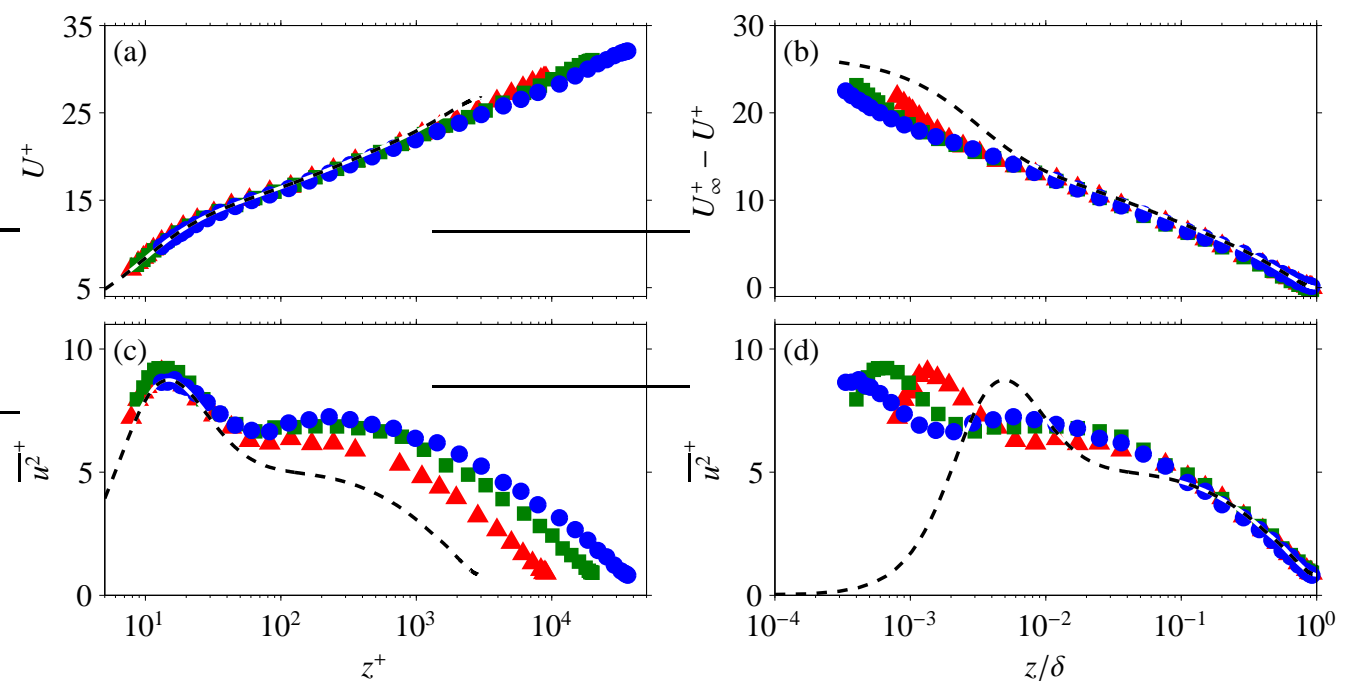

Figure 2: Streamwise velocity statistics from the pipe experiments. Panels (a,b) and (c,d) show the mean and variance profiles, respectively. The symbols denote different $R e$ cases, i.e. $\Delta: R e_{\tau} \approx$ $10000\left(l^{+}=11\right), \boldsymbol{\square}: R e_{\tau} \approx 22000\left(l^{+}=24\right)$ and $\bullet: R e_{\tau} \approx 40000\left(l^{+}=44\right)$, while the dashed (-- ) lines correspond to the statistics from Ahn et al. (2015) at $R e_{\tau} \approx 3000\left(2 \pi R^{+} / N_{\theta}=6\right.$, where $N_{\theta}$ is the number of grids in the DNS along the azimuthal direction).

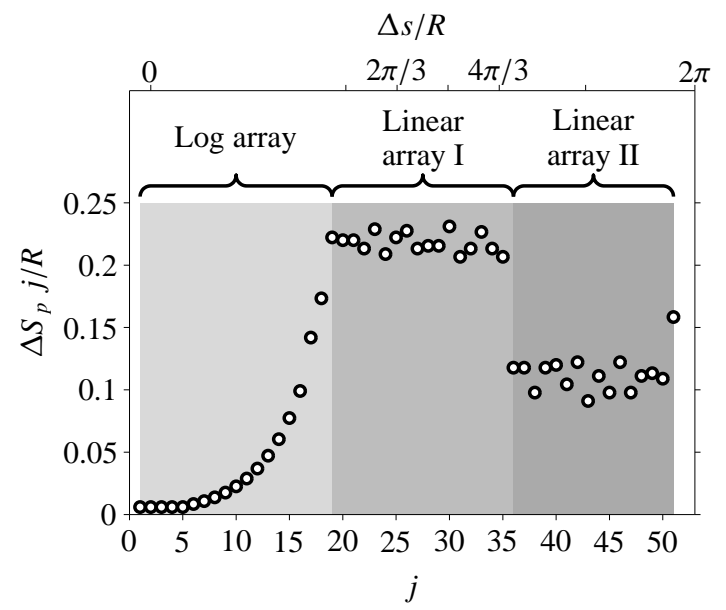

Figure 3: Azimuthal spacing, $\Delta S_{p}$, between the adjacent sensors in the hot-film array, for the pipe experiment.

mismatch in the $z$ locations between the hot-wire probe and Pitot-static tube during calibration) since only relative changes in the skin friction and $u$ velocity are considered.

Senflex hot-film sensor arrays from Tao systems are used to measure the skin friction. The three configurations depicted in figure 1(a) are used: a log array, a linear array I and a linear array II. The closely spaced sensors around the hot-wire traverse plane capture the smallscale contributions to the two-point statistics between $u$ and $u_{\tau}$, while the coarsely spaced sensors capture the large-scale contributions (these lose coherence much more gradually with increasing azimuthal offset, $\Delta s$ ). The azimuthal offsets of the sensors in the log array increase 
logarithmically from $\Delta s / R \approx 0.006$ to 0.22 . Following the log array, the spacing in the linear array I is maintained at $\Delta s / R \approx 0.22$ from the 20th to 35th sensor. This spacing is halved to form linear array II. Furthermore, the sensors $S_{p} 1-17$ are printed on to a single substrate, allowing the azimuthal offsets to be precisely set, while the remainder are manually positioned and the offsets recorded. The hot-wire is set to traverse nominally in the same plane as $S_{p} 2$. Figure 3 shows the azimuthal offset between the adjacent hot-film sensors for the pipe experiment, where $\Delta S_{p} j$ denotes the spacing between the $j$ and $j+1$ th sensors. To calibrate the hot-film sensors, the mean voltage outputs are recorded against the mean shear stress, obtained from measuring the axial pressure drop in the pipe before and after each experiment. The calibration curve for the hotfilms follows a similar technique described for the hot-wire previously. However, since the hotfilms are fixed in space, the voltage drift over time can be estimated throughout the measurement without the need for interpolation. Each of the 51 hot-films is operated by individual Melbourne University Constant Temperature Anemometer (MUCTA), Dantec StreamLine, AA labs AN1003 and Dantec Multichannel Constant Temperature Anemometers, each set to an overheat ratio of 1.05 .

To ensure that the 51 hot-film voltages and the hot-wire voltage are acquired simultaneously, a common clock signal is utilised across five data translation DT9836 data acquisition boards used for analogue-to-digital conversion. To accommodate a faster temporal response expected from the hot-wire, it is sampled at a higher frequency than the hot-films. Furthermore, the two frequencies are chosen such that their ratio is an integer, thus allowing the hot-wire signal to be downsampled at the hot-film sampling frequency. In addition, a common hot-film voltage is shared between the five acquisition boards which is then used to verify the synchronicity of the acquisition. The hot-wire and hot-film details are summarised in table 2.

\subsection{Boundary layer flow}

The boundary layer dataset is from Hutchins et al. (2011), which was acquired at the high Reynolds number boundary layer facility located at the University of Melbourne. The boundary layer thickness at the measurement location is approximately $0.33 \mathrm{~m}$. As with the pipe experiments, this large $\delta$ allows access to the high $R e$ regime using conventional techniques (see figure $1 \mathrm{~b}$ for comparison against the pipe facility in physical units). Consistent with the pipe measurements, a $2.5 \mu \mathrm{m}$ platinum Wollaston wire with a length to diameter ratio of 200 is used as a velocity sensor, while ten Dantec 55R47 glue-on-type hot-film sensors are used to measure the skin friction. The spanwise spacing between all ten hot-films is kept constant $\left(\Delta S_{b} 1-9 \approx 0.08 \delta\right)$ and the hot-wire is located nominally above the sixth sensor, $S_{b} 6$, as illustrated in figure 1(c). The hot-films and hot-wire are operated using AA labs AN1003 CTA systems with overheat ratios of 1.05 and 1.8, respectively. The hot-wire sensor is calibrated in the free stream against a Pitot-static tube before and after the measurement and linearly interpolated based on the working section temperature recorded during the measurement. The hot-film sensors are calibrated via an empirical relationship between the free-stream velocity and the mean wall-shear stress. For further details, the reader is referred to Hutchins et al. (2011).

\section{Basic features of the data}

The limited temporal response of a typical hot-film sensor, combined with spatial averaging effects due to finite sensor size, precludes full resolution of the small-scale skin friction contributions. This is evident when the ratio between the standard deviation and mean measured skin friction, $\sigma(\tau) / \bar{\tau}$, is calculated. Direct numerical simulation and resolved experiments indicate $\sigma(\tau) / \bar{\tau} \approx 0.3-0.4$ with a weak $\operatorname{Re}$ dependency at high $\operatorname{Re}$ (de Silva et al. 2014), while the ratio reported by hot-film experiments is typically approximately 0.1 (Alfredsson et al. 1988). Despite the inability of hot-film sensors to accurately capture the amplitudes of the skin friction 


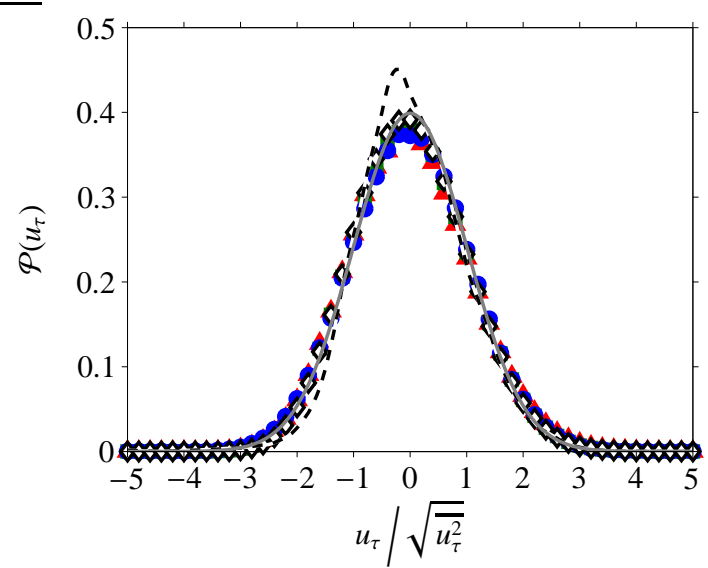

Figure 4: Probability density function of fluctuating friction velocity, $\mathcal{P}\left(u_{\tau}\right)$. The solid symbols correspond to hot-film dataset from a pipe flow at $R e_{\tau} \approx 10000(\Delta), 22000$ (匹) and 40000 $(\bullet)$. Further, the open symbol $(\diamond)$ and dashed line (---) show $\mathcal{P}\left(u_{\tau}\right)$ from a boundary layer flow at $R e_{\tau} \approx 14000$, obtained using hot-films and high-magnification PIV (de Silva et al. 2014), respectively. The solid line (-) indicates the standard normal distribution.

fluctuations, they still retain many of the characteristic features of the resolved skin friction measurements (Alfredsson et al. 1988). Hence, in this paper, we will report fluctuating friction velocities relative to the measured standard deviation, rather than the absolute value.

\subsection{Single- and two-point statistics}

Figure 4 shows the probability density function (pdf) of friction velocity, $\mathcal{P}\left(u_{\tau}\right)$, from the current dataset, where $u_{\tau}=\sqrt{\tau / \rho}$ and $\rho$ the density of the fluid. As is evident from the figure, the data merge across the range of Reynolds numbers examined, once $u_{\tau}$ has been normalised by its standard deviation. Furthermore, this agreement extends to boundary layer flows where the friction velocity is measured using hot-films $(\diamond)$. Also note that, in contrast to pdf of wall-shear stress, $\mathcal{P}(\tau)$, which is positively skewed (de Silva et al. 2014), $\mathcal{P}\left(u_{\tau}\right)$ is near symmetric. The missing contributions from small scales in the hot-film measurements are primarily associated with the unresolved skin friction contributions from the near-wall dynamics. In contrast, the wellresolved large-scale contributions are associated with larger structures residing in the logarithmic region. Since the $u$ velocity is less Gaussian in the near wall $\left(z^{+} \lesssim 20\right)$ than in the logarithmic region, this leads to a more Gaussian pdf when compared to a better-resolved dataset (---) obtained using high-magnification particle image velocimetry (PIV) techniques (de Silva et al. 2014) (e.g. the skewness and kurtosis of $u_{\tau}$ recorded by the hot-film are 0.15 and 2.9 respectively, in contrast to -0.55 and 7.2 obtained from the high-magnification PIV dataset).

Figure 5(a,b) shows the two-point correlation of $u_{\tau}, R_{u_{\tau} u_{\tau}}$ from the current dataset as functions of longitudinal (streamwise) and transverse offsets, respectively. It should be noted that the streamwise offset, $\Delta x$ in (a) is obtained through use of Taylor's frozen turbulence hypothesis (Taylor 1938), where a convection velocity, $U_{c}$, is used to convert the hot-film signal from the temporal to the spatial domain. Following work of Hutchins et al. (2011), who examined spacetime correlation of two hot-film sensors separated in $x$, the convection velocity for the large-scale $u_{\tau}$ fluctuations is estimated to be the mean velocity at the $z$ location where the very-large-scale $u$ fluctuations are strongest (i.e. the large-scale $u_{\tau}$ features convect at the same rate as the verylarge-scale $u$ fluctuations residing in the logarithmic region). This wall height approximately corresponds to $z^{+}=\sqrt{15 R e_{\tau}}$ according to Mathis et al. (2009), hence based on the logarithmic 

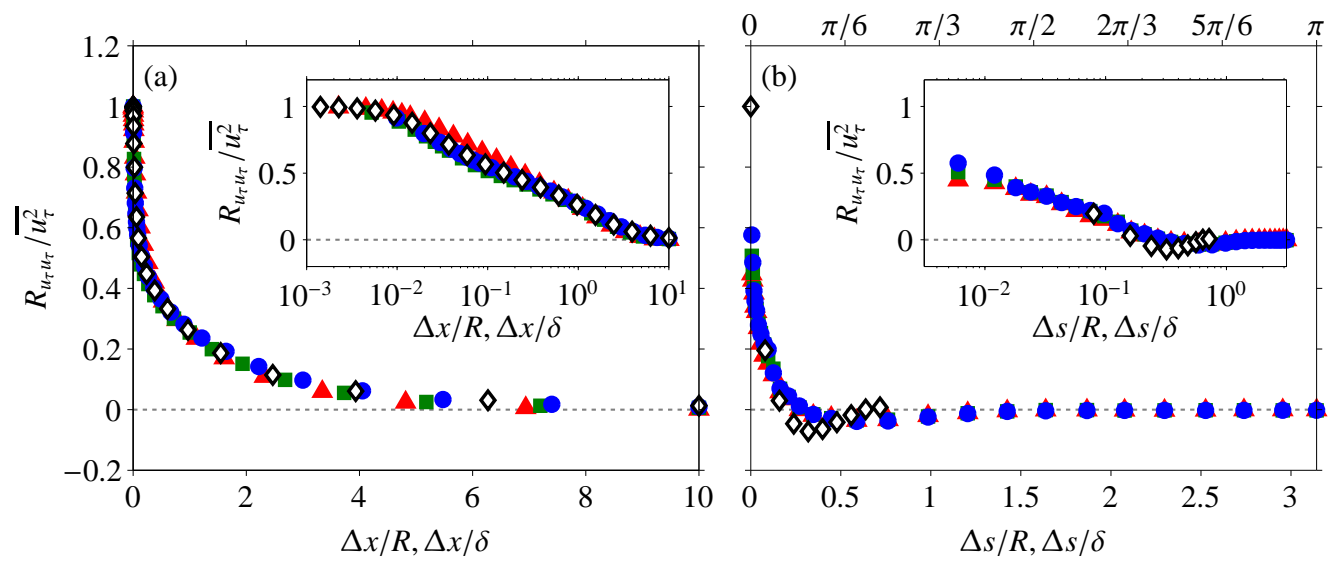

Figure 5: Two-point correlation of fluctuating friction velocity, $R_{u_{\tau} u_{\tau}}$. The correlation coefficients are shown as functions of (a) the longitudinal $\left(R_{u_{\tau} u_{\tau}}(\Delta x, 0)\right)$ and (b) transverse $\left(R_{u_{\tau} u_{\tau}}(0, \Delta s)\right)$ offsets. The solid symbols correspond to pipe flow at $R e_{\tau} \approx 10000(\Delta), 22000$ (匹) and 40000 $(\bullet)$, while the open symbols $(\diamond)$ correspond to boundary layer flow at $R e_{\tau} \approx 14000$. For the insets, a logarithmic scale is used for the displacements, $\Delta x$ and $\Delta s$, between the two points.

law we obtain

$$
\frac{U_{c}}{U_{\tau}}=\frac{1}{\kappa} \ln \sqrt{15 R e_{\tau}}+A,
$$

where $\kappa=0.39$ and $A=4.3$ are the Kármán constant and the logarithmic intercept for canonical wall-bounded flows (Marusic et al. 2013). Although the frozen turbulence hypothesis is known to breakdown for the small scales in the near-wall region (del Álamo \& Jiménez 2009; Piomelli et al. 1989), since the hot-film sensors only resolve large-scale contributions, it is a reasonable approximation for the signals obtained from these sensors. For the transverse offset, the correlation is calculated from two sensors separated by distance $\Delta s$ in the azimuthal and spanwise directions for the pipe and boundary layer flows, respectively. It should be also noted that, while only selective $\Delta x$ locations (corresponding to a specific $\Delta t$ offset in the $u_{\tau}$ time series) are shown in figure 5(a), $\Delta s$ locations from each hot-film pair are shown in figure 5(b).

As is evident from figure 5(a,b), good agreement is observed for $R_{u_{\tau} u_{\tau}}$ from the current dataset $(\Lambda, \square$ and $\bullet$ ) for both streamwise and azimuthal offsets, when normalised by the outer length scale. This is because the correlations beyond $\Delta x / R, \Delta s / R>O(0.1)$ are dominated by the largescale motions which scale with the outer length scale. Similar results have been observed for twopoint correlations of streamwise velocity (Hutchins \& Marusic 2007a). However, for small $\Delta x$, the collapse of $R_{u_{\tau} u_{\tau}}$ across different $R e$ is no longer expected when normalised by the outer length scale, since the scale separation between the viscous and inertially dominated scales increases with increasing $R e$. This is also evident in the experimental data when a logarithmic scale is used for $\Delta x$ (e.g. figure 5a inset), where $R_{u_{\tau} u_{\tau}}$ at $\Delta x / R \sim 0.1$ no longer exhibits a collapse. Notably, the collapse of $R_{u_{\tau} u_{\tau}}$ for a large $\Delta x$ extends to the boundary layer case $(\diamond)$ when the streamwise offset distance is normalised by its respective outer length scale, as seen in figure 5(a). However, figure 5(b) shows that unlike the streamwise offset, $R_{u_{\tau} u_{\tau}}$ from the pipe and boundary layer flows exhibit discernible differences in the transverse direction even after the offset has been normalised by the outer length scale. A similar difference between internal (channel and pipe) and external (boundary layer) flows is reported by Monty et al. (2007) for correlations of $u$. They attribute the differences to variations in the coherent structures that extend beyond the logarithmic region $(z / R, z / \delta>0.15)$. In internal flow, these coherent structures are persistent, 

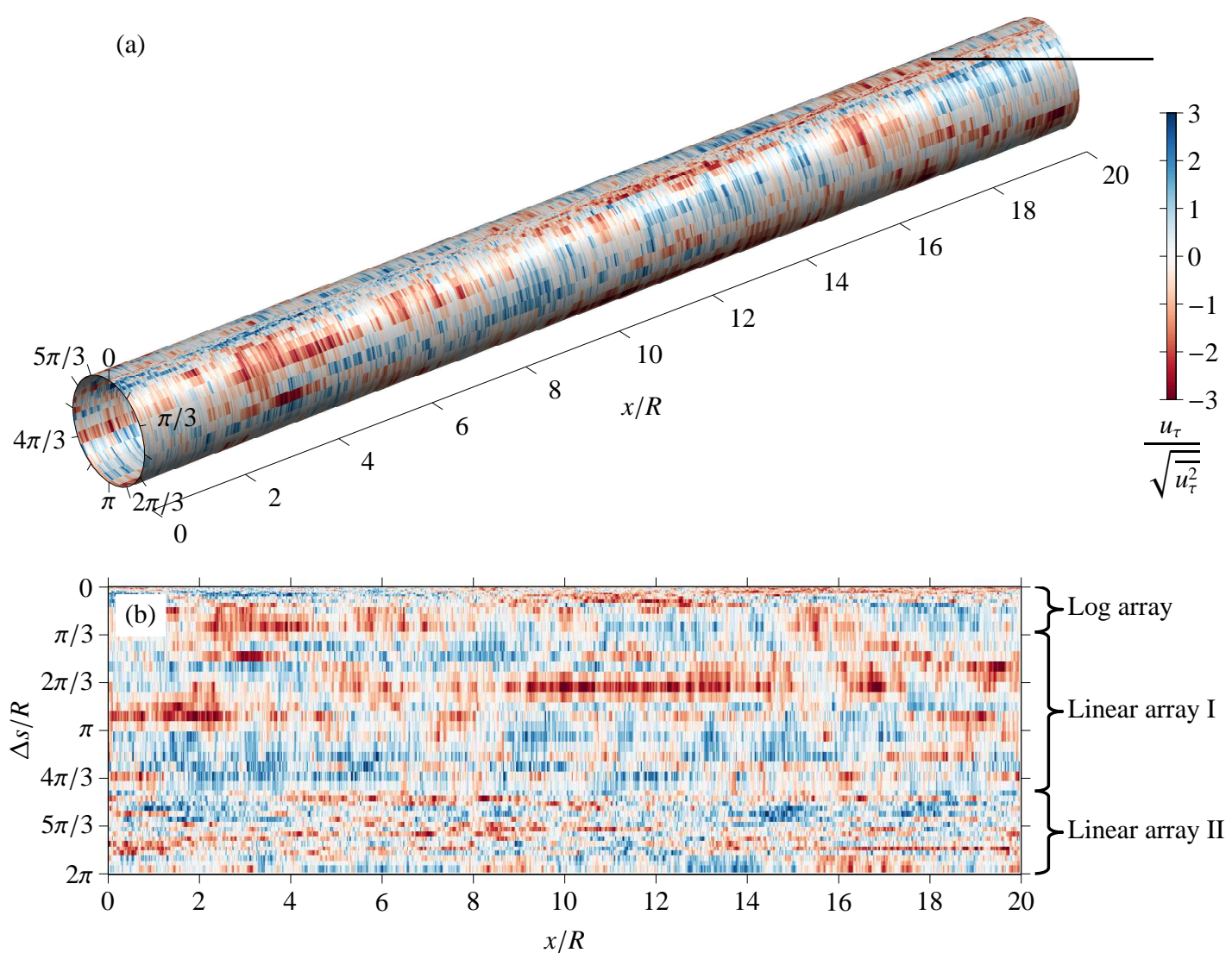

Figure 6: Instantaneous fluctuating friction velocity, $u_{\tau}$, recorded by hot-film sensors at $R e_{\tau} \approx$ 40000 . (a) Shows a three-dimensional view of $u_{\tau}$ along a pipe surface, while its projection on to a flat plane is shown in (b).

while in the external flow they breakdown more readily into smaller eddies, resulting in $R_{u_{\tau} u_{\tau}}$ decreasing faster in the transverse direction. As a result, the anti-correlated region where $R_{u_{\tau} u_{\tau}}$ is most negative shifts from $\Delta s / R \approx 0.6$ to $\Delta s / \delta \approx 0.4$ between the internal and external flows (see figure 5b), replicating the $R_{u u}$ behaviour observed in the logarithmic region (Sillero et al. 2014).

\subsection{Instantaneous visualisations}

Figure 6 shows an instantaneous view of the friction velocity from the current dataset, at $R e_{\tau} \approx$ 40000 . Since the spacing between the hot-films is $0.1 R-0.2 R$ for the two linear arrays (covering almost $85 \%$ of the total pipe circumference), the $R$-scaled features dominate the instantaneous view. These $R$-scaled features are associated with very-large-scale motions (VLSMs) (Kim \& Adrian 1999) that largely reside in the logarithmic region and whose influence extends down to the wall (Hutchins \& Marusic 2007a). Thus, these footprints impose large-scale $u_{\tau}$ features that meander and extend over $O(10 R)$ in the streamwise direction with a width of $O(R)$ in the azimuthal direction (Monty et al. 2007). 


\section{Spectral coherence with respect to the wall}

In order to assess the scale-based linear coupling between the streamwise velocity fluctuations, $u$, and the fluctuating friction velocity at the wall, we employ a spectral coherence analysis. We use the spectral domain equivalent of a physical two-point correlation, known as the linear coherence spectrum, to compute the correlation per scale (Baars et al. 2017). The coherence spectrum, $\gamma_{L}^{2}$, is defined as

$$
\gamma_{L}^{2}\left(\Delta s, z ; \lambda_{x}\right)=\frac{\left|\left\langle\widehat{u}\left(z ; \lambda_{x}\right) \widehat{u}_{\tau}^{*}\left(\Delta s ; \lambda_{x}\right)\right\rangle\right|^{2}}{\left\langle\left|\widehat{u}\left(z ; \lambda_{x}\right)\right|^{2}\right\rangle\left\langle\left|\widehat{u}_{\tau}\left(\Delta s ; \lambda_{x}\right)\right|^{2}\right\rangle}=\frac{\left|\phi_{u u_{\tau}}\left(\Delta s, z ; \lambda_{x}\right)\right|^{2}}{\phi_{u u}\left(z ; \lambda_{x}\right) \phi_{u_{\tau} u_{\tau}}\left(\Delta s ; \lambda_{x}\right)},
$$

where, $\widehat{u}\left(z ; \lambda_{x}\right)=\mathcal{F}[u(z)]$ denotes the Fourier transform of $u(z)$ in the $x$ direction; while the asterisk $(*)$, angle brackets $(\langle\rangle)$ and vertical bars $(||)$ designate the complex conjugate, ensemble averaging and modulus, respectively. Thus, $\phi_{u u_{\tau}}$ corresponds to the cross-spectrum between $u$ and $u_{\tau}$, while $\phi_{u u}$ and $\phi_{u_{\tau} u_{\tau}}$ denote the $u$ - and $u_{\tau}$-energy spectra, respectively. The denominator of (4.1) is such that $\gamma_{L}^{2}$ normalisation occurs per-scale (and hence provides the square of a scalespecific correlation coefficient) and for all scales is bounded within $0 \leqslant \gamma_{L}^{2} \leqslant 1$. This property of $\gamma_{L}^{2}$ is especially useful when using the hot-film data, as its scale-dependent energy attenuation does not affect a per-scale normalised correlation (Baars et al. 2017; Bendat \& Piersol 2010).

As described in $\S 3.1$, temporal data are obtained from both hot-film and hot-wire sensors. Thus, $\gamma_{L}^{2}$ is calculated in the frequency domain and converted to $\lambda_{x}$ by invoking Taylor's hypothesis. Here, the local mean velocity at $z$ (corresponding to the wall-normal location where the $u$ velocity is acquired) is used as the convection velocity. This procedure results in coherence spectra that agree well with that calculated directly from DNS (Baars et al. 2017), with the exception of the near-wall small scales, where the assumed convection velocity no longer holds (del Álamo \& Jiménez 2009; Piomelli et al. 1989). As the focus of this paper is on the logarithmic region, the errors associated with Taylor's hypothesis will not affect the conclusions drawn.

\subsection{Comparisons between pipe and boundary layer flows}

Figure 7(a,(b)) shows $\gamma_{L}^{2}$ between the streamwise velocity, $u$, and the friction velocity, $u_{\tau}$, as a functions of wavelength $\lambda_{x}$. The solid circles (๑) in both (a) and (b), correspond to the coherence spectrum for the pipe flow when $u$ is acquired at $z / R \approx 0.01$ and is measured directly above $u_{\tau}$, as illustrated in (c). Similarly, the empty circles $(O)$ correspond to the coherence spectrum for the boundary layer flow, where $u$ is measured $0.01 \delta$ above $u_{\tau}$.

As stated in the introduction, one of the aims of this paper is to extend the observation of Baars et al. (2017), which supports wall-bounded turbulence obeying the AEH. Figure 8 shows a sketch illustrating relative scales in the AEH, where a hierarchy of self-similar structures is used to represent the logarithmic region in a wall-bounded flow. The size of each hierarchy level is shown in a different colour, and the hierarchy follows a geometric progression with a common ratio of 2 up to a height equal to the outer length scale. In this schematic the population density halves in the $x$ and $y$ dimensions for each increment in the hierarchy level. Thus, in figure 8 a total of four hierarchy levels are shown, and eddies at each level have an extent in the $x, y$ and $z$ directions characterised here by $\mathcal{L}_{i}, \mathcal{W}_{i}$ and $\mathcal{H}_{i}$, respectively. Here, subscript $i=1-4$ is used to denote each hierarchy level, with the smallest and largest following $\mathcal{H}_{1} \sim O\left(v / U_{\tau}\right)$ and $\mathcal{H}_{4} \sim O(\delta)$, respectively (Perry \& Chong 1982). It should be noted that in reality, these structures are forward inclined relative to the flow direction (Robinson 1991). Since, however, only the magnitude of the coherence (and not the phase) is considered here, this detail is not relevant to the present discussion. Now, consider probes placed at $\mathbf{\square}$ (measuring $u_{\tau}$ ) and $\bigcirc$ (measuring $u$ ) as illustrated in figure 8(a), leading to recorded signals from these probes as in figure 8(b). It is therefore evident that the signal from the $\bigcirc$ sensor misses contributions from the smallest eddies 

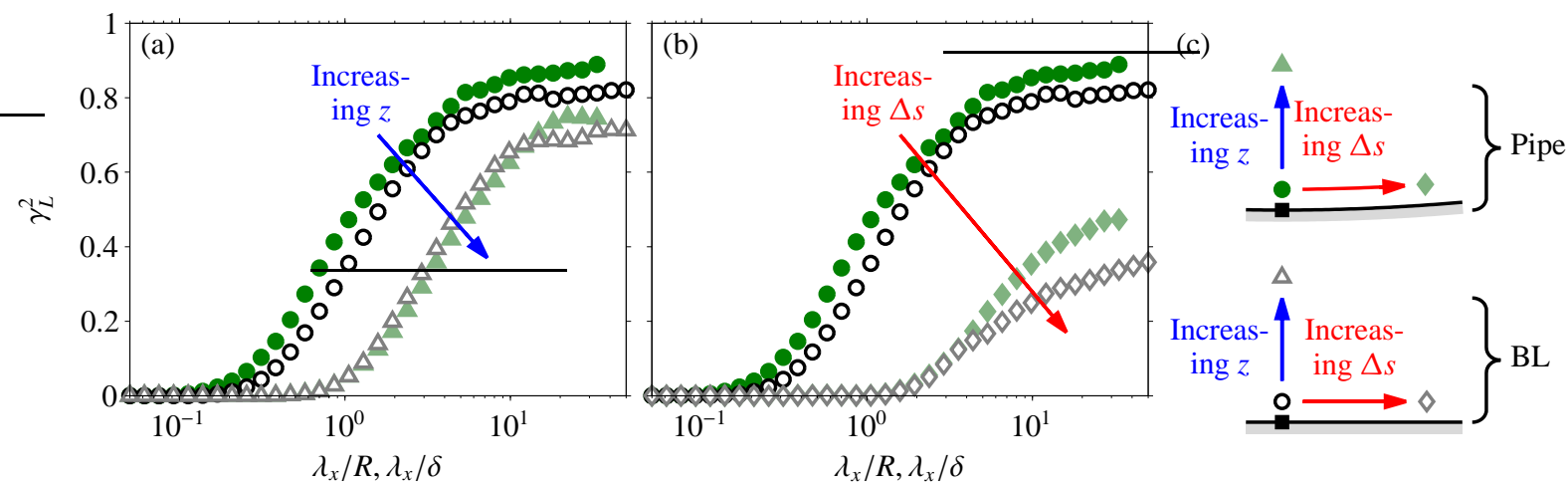

Figure 7: Coherence spectrum, $\gamma_{L}^{2}$, between the streamwise velocity, $u$, and the friction velocity, $u_{\tau}$, as a function of wavelength $\lambda_{x}$. The solid $(\bullet, \Delta, \diamond)$ and open $(\bigcirc, \Delta, \diamond)$ symbols respectively correspond to the pipe and boundary layer (BL) flows. Also, the pipe and boundary layer flows are at $R e_{\tau} \approx 22000$ and 14000 , respectively. (a) Two wall-heights $z / R, z / \delta \approx 0.01(\bullet, \bigcirc)$ and $0.07(\Delta, \Delta)$ at $\Delta s \approx 0$; and (b) two hot-film offsets $\Delta s / R, \Delta s / \delta \approx 0(\bullet, \bigcirc)$ and $0.07(\diamond, \diamond)$ at $z / R, z / \delta \approx 0.01$ are shown. (c) Schematic of hot-wire and hot-film positions used to generate (a) and (b), where $\mathbf{m}$ symbol denotes the reference skin friction sensor.
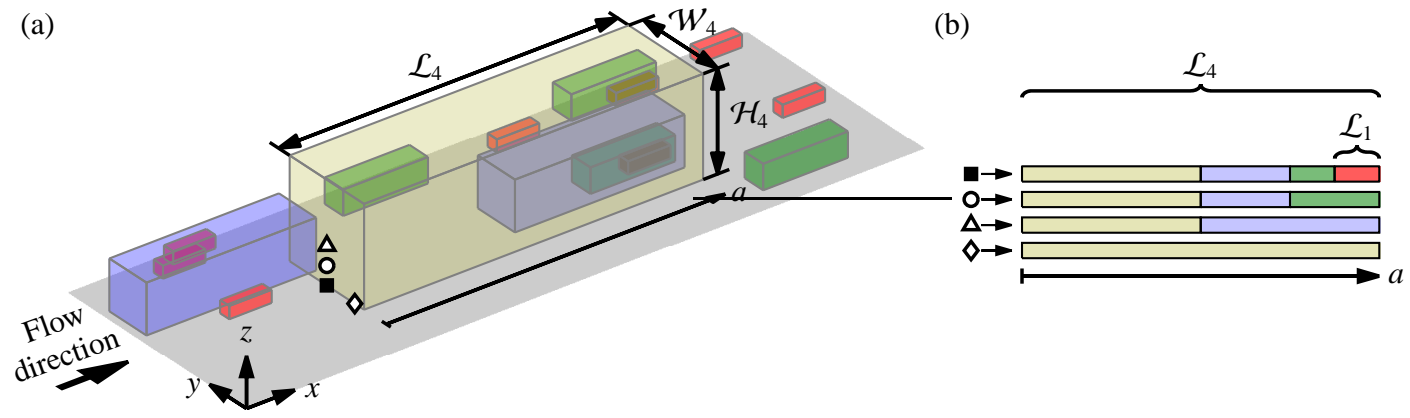

Figure 8: (a) Schematic showing a hierarchy of attached eddies used to model wall-bounded flows. Here, four hierarchy levels are shown, where a volume of influence from each is represented by a differently coloured cuboid, while the symbols indicate probe locations. (b) The contribution to $u$ or $u_{\tau}$ signals at four locations (as indicated by corresponding symbols in a) over a streamwise distance $a ; \mathcal{L}_{i}, \mathcal{W}_{i}$ and $\mathcal{H}_{i}$ denote the streamwise, spanwise and wall-normal extent of an $i$ th hierarchy level eddy.

when compared to the signal from the $\mathbf{a}$ sensor. In contrast, the large-scale component of these signals is still mostly coherent, leading to a steady decay in $\gamma_{L}^{2}$ from $\sim 0.8$ to 0 with decreasing $\lambda_{x}$, as observed in figure $7(\mathrm{a}, \mathrm{b})$.

Figure 7(a) shows the effect of increased separation between $u$ and $u_{\tau}$ in the $z$ direction. Here, $\Delta$ symbols correspond to a case where $u$ is obtained at a higher $z$ location than the $\bigcirc$ symbols as illustrated in figure 7(c). A decrease in $\gamma_{L}^{2}$ is observed at all scales in both the pipe and boundary layer flows with increased $z$ separation. In terms of the $\mathrm{AEH}$, this relates to a reduction in the number of common members of the hierarchy encountered with increased $z$, as shown by idealised $u$ signals at $\bigcirc$ and $\Delta$ in figure 8(b). Similarly, figure 7(b) shows the effect of increased separation between $u$ and $u_{\tau}$ in the transverse direction, with the $\diamond$ symbol corresponding to a case where $u$ is obtained with an azimuthal/spanwise offset, $\Delta s$, compared to the $\bigcirc$ symbols 
Pipe

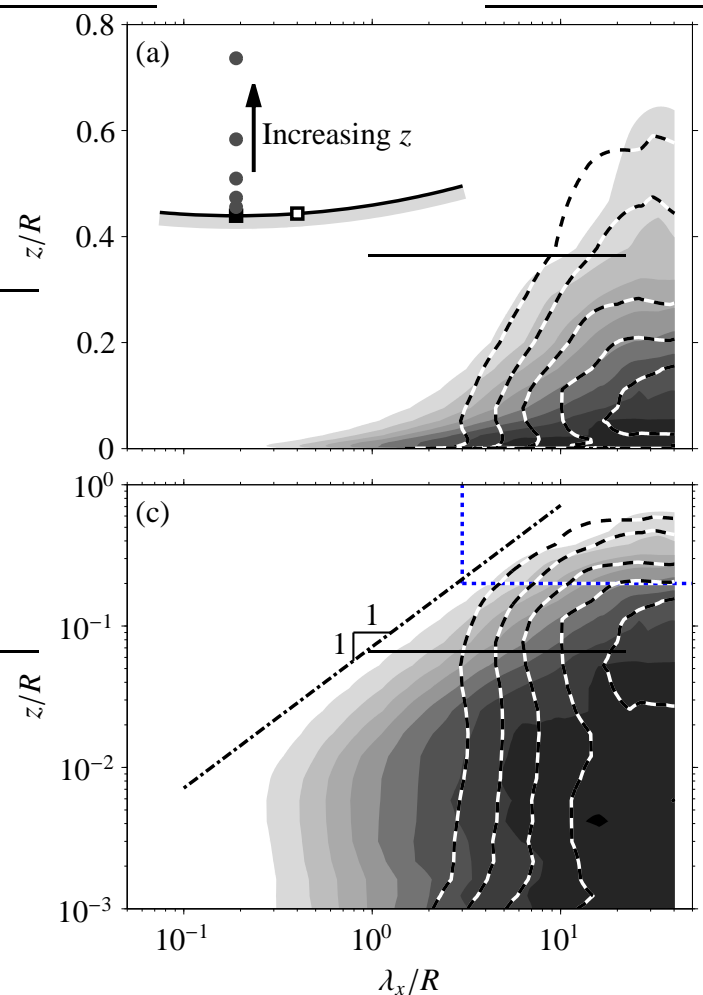

Boundary layer

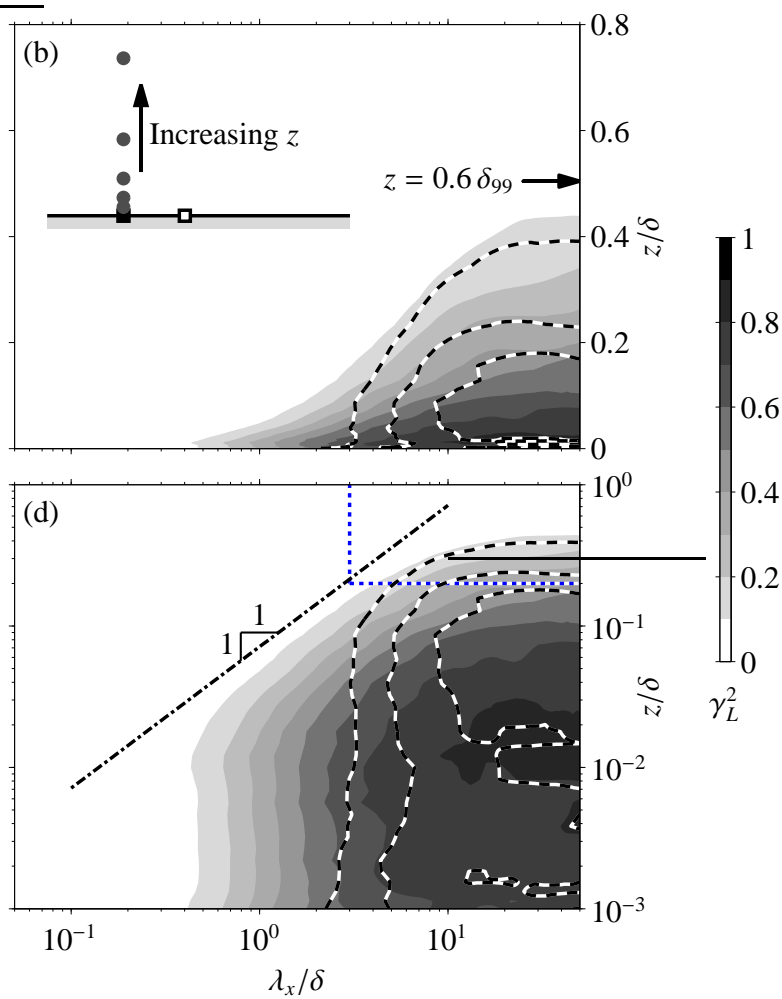

Figure 9: Coherence spectrum between $u_{\tau}$ and $u, \gamma_{L}^{2}$, as a function of wavelength, $\lambda_{x}$, and wallnormal location, $z$, where $u$ is measured. (a,c) Pipe $R e_{\tau} \approx 22000$ and (b,d) boundary layer $R e_{\tau} \approx 14000$ flows, where $z$ is shown in linear and logarithmic scale on the top $(\mathrm{a}, \mathrm{b})$ and bottom $(\mathrm{c}, \mathrm{d})$ rows, respectively. The grey scale contours correspond to coherence when the hot-film sensor ( $\square$ ) is at an offset of $\Delta s \approx 0$ from the hot-wire ( $\bullet$, while the line contours show coherence at an offset of $\Delta s / R, \Delta s / \delta \approx 0.07$ (口). Both line and grey scale contours are at levels 0.1:0.1:0.9, and the dash-dotted lines indicate the $\lambda_{x}=R_{x z} z$ relationship, where $R_{x z}=14$ corresponds to empirically observed aspect ratio between $\lambda_{x}$ and $z$ for the self-similar hierarchy.

as indicated in figure 7(c). Again, a decrease in $\gamma_{L}^{2}$ at all scales is observed in both the pipe and boundary layer flows with increased $\Delta s$ separation, but the decrease is much more severe compared to that observed for an equivalent increase in $z$. As discussed later, these differences can also be explained by considering an idealised model based on the AEH.

Figure 9 shows coherence spectra as functions of $\lambda_{x}$ and $z$. The left $(\mathrm{a}, \mathrm{c})$ and right $(\mathrm{b}, \mathrm{d})$ figures correspond to $\gamma_{L}^{2}$ for the pipe and boundary layer flows, respectively. The top (a,b) and bottom (c,d) figures correspond to the same coherence spectra, but $z$ is shown in the linear and logarithmic scales, respectively. It should be noted that there exists a slight discrepancy in $R e_{\tau}$ between the two flows (22000 for the pipe compared to 14000 for the boundary layer), however across this range, we expect the $R e_{\tau}$ effects on the large-scale features to be minimal when scaled by the outer length scales (Hutchins \& Marusic 2007a,b). Consequently, here we present $\lambda_{x}$ and $z$ normalised by $R$ and $\delta$ respectively for the pipe and boundary layer flows.

From figures 9(a) and (b), it is evident that the large-scale component of $u$ motions remains coherent over a larger $z$ extent for the pipe compared to the boundary layer flow when normalised by the corresponding outer length scale, in agreement with the observations of Monty et al. 
(2007). In an internal flow such as a pipe, instances exist where a large-scale $u$ event remains coherent over a $z$ extent larger than $R$ (i.e. penetrates beyond the centreline while starting at the wall) (Sillero et al. 2014), while $\delta$ for the boundary layer flow is closer to the outermost edge of the turbulent region, and beyond which only a non-turbulent flow exists (Chauhan et al. 2014). This mismatch in the outer length scale used for the normalisation relative to the size of the largest coherent motions presumably leads to some of the differences observed between the coherence spectra from the pipe and boundary layer flow in the wake region. It should be noted that, a choice of $\delta_{99}$ (i.e. the $z$ location where $U=0.99 U_{\infty}$ ) as the outer length scale for the boundary layer instead of $\delta$, yields a better agreement in the wake region as evident from the $z=0.6 \delta_{99}$ position indicated on figure 9(b). Despite the modification to the choice of outer length scale, the differences in the $u$-spectra between the internal and external flows still persist, since as demonstrated by Monty et al. (2009), it is not possible to achieve a full merging of the $u$ spectra across the entire large wavelength range, irrespective of the outer length-scale definition chosen.

Another potential source of difference between the internal and external flows in figure $9(a, b)$, may be due to use of Reynolds decomposition in obtaining the $u$ fluctuations. While this is a standard practice, if multiple states with different mean exist in a flow (such as turbulent and nonturbulent regions), all states are reduced to a single common mean (Kwon et al. 2016). Hence, the use of Reynolds decomposition can exacerbate observed differences between internal and external flows under outer scaling, as demonstrated by Kwon (2016), who improved the collapse of the two-point correlation of a boundary layer and a channel using an alternate decomposition that separated the turbulent and quiescent core/non-turbulent regions. However, it should also be noted that both choices for the $u$ fluctuations provide similar results in the logarithmic and near-wall regions as the turbulent portion dominates at these $z$ locations.

The grey scale contours in figure 9 correspond to the coherence spectra between $u_{\tau}$ and $u$ for $\Delta s \approx 0$, while the line contours show $\gamma_{L}^{2}$ when an azimuthal/spanwise offset of $\Delta s / R, \Delta s / \delta \approx$ 0.07 exists between the hot-film and hot-wire (see inset, symbols $\square$ and $\bullet$ ). Based on the attached eddy model illustrated in figure 8, we would expect the two hot-film sensors to share large-scale $u_{\tau}$ features since they share a common footprint from eddies that span across both sensors. This is indeed reflected in figure 9 , where the two coherence spectra at $\Delta s=0$ and $\Delta s / R, \Delta s / \delta \approx 0.07$ show good agreement for $\lambda_{x} / R, \lambda_{x} / \delta>3$ and $z / R, z / \delta>0.2$ (top-right regions encapsulated by the dotted lines in figure $9 \mathrm{c}, \mathrm{d})$. As the effect of the differing outer boundary conditions between the internal and external flow is diminished closer to the wall, a good agreement is also observed between the pipe and boundary layer flows (see figure $9 \mathrm{c}, \mathrm{d}$ ) in the logarithmic region. This includes the iso-contours of the coherence spectra following a $\lambda_{x} \sim z$ scaling (shown as dot-dashed lines). This behaviour is consistent with the AEH, which assumes that the coherent structures are self-similar and scale with the distance from the wall (Townsend 1976). The dash-dotted lines in figure 9(c) and (d) show the relationship $\lambda_{x}=R_{x z} z$, where $R_{x z}=14$ corresponds to $\lambda_{x}$ where wall-shear stress and $u$ velocity starts to exhibit coherence in the logarithmic region (Baars et al. 2017). Thus, these features are much longer in $x$ compared to their $z$-extent (i.e. $R_{x z} \gg 1$ ), indicating that the coherent motions in $u$ are likely associated with multiple streamwise aligned eddies travelling in a packet (Adrian et al. 2000), rather than a single isolated eddy.

Here, we propose to characterise the dimensions $(\mathcal{L} \times \mathcal{W} \times \mathcal{H})$ of the self-similar attached eddies based on $\gamma_{L}^{2}$ iso-contours, following Baars et al. (2017). Note that, while the attached eddies are confined in the wall-normal direction by the presence of the wall, no such confinement exists in the spanwise direction leading to differences in how $\gamma_{L}^{2}$ approaches zero as a function of $\Delta s$ and $z$ (e.g. figure 7). As an example, consider the idealised, discrete eddy, scenario shown in figure 8, when an eddy passes over a reference skin friction sensor. As illustrated in figure 10(b), this requires the reference skin friction sensor (denoted by symbol $\mathbf{a}$ ) to be located within the 
(a)
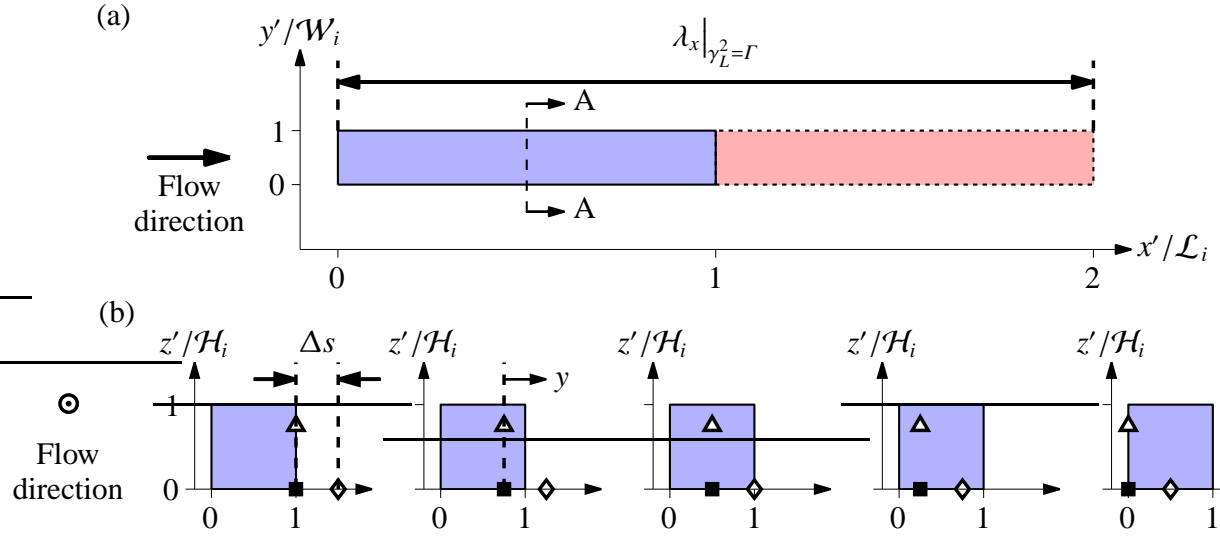

(c)

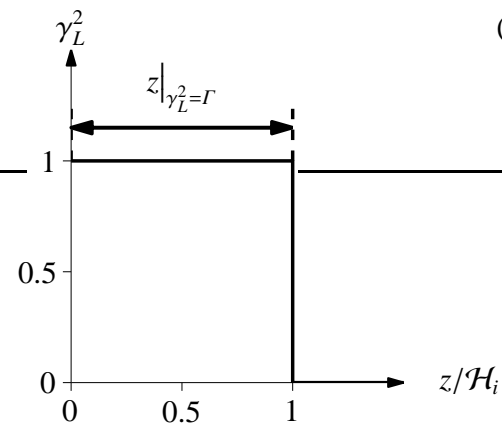

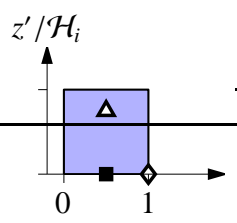
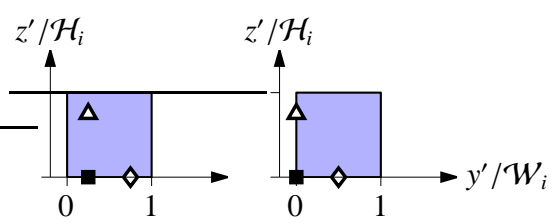

(d)

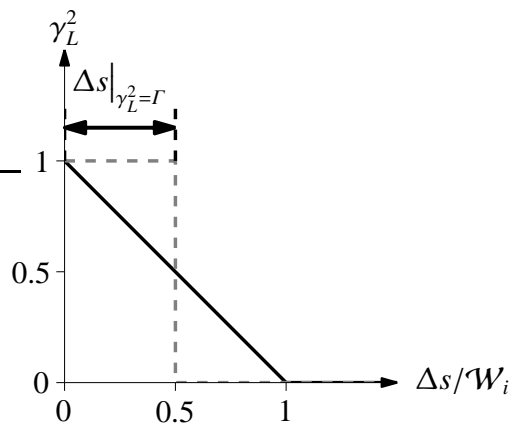

Figure 10: An illustration of size of an $i$ th hierarchy level eddy, $\mathcal{L}_{i} \times \mathcal{W}_{i} \times \mathcal{H}_{i}$, extracted based on $\lambda_{x}, \Delta s$ and $z$ where $\gamma_{L}^{2}=\Gamma$ ( $\Gamma$ is a threshold). (a) Wall-parallel ( $x-y$ plane) view of volume of influence and (b) cross-plane ( $y-z$ plane) view (along A-A in figure 10a) showing different scenarios where the volume of influence passes over a reference skin-friction sensor $(\square)$. The blue and red regions in (a) and (b) denote negative and positive $u$, respectively, while $x^{\prime}, y^{\prime}$ and $z^{\prime}$ are coordinates relative to the eddy. The solid lines in $(\mathrm{c}, \mathrm{d})$ show idealised coherence as a function of offset distances $z$ and $\Delta s$ between the two sensors in the wall-normal and spanwise directions, respectively. The dashed line in (d) shows an equivalent step-like profile proposed.

low shear stress footprint of the eddy with an equal probability. For a case when the shear stress and velocity sensors are spanwise aligned, the velocity recorded at a wall-normal offset $z, u(z)$, is perfectly correlated with the wall-shear stress sensor $\left(\gamma_{L}^{2}=1\right)$ if $z<\mathcal{H}_{i}$, otherwise the two signals remain uncorrelated $\left(\gamma_{L}^{2}=0\right)$, regardless of the spanwise position of the eddy, leading to a step like $\gamma_{L}^{2}$ as a function of $z$ as shown in figure 10(c). In contrast, for a case when $\Delta s \neq 0$, the likelihood that influence of the eddy extends to $y=\Delta s$ is no longer equal to unity even when $z<\mathcal{H}_{i}$. This is because unlike the $\Delta s=0$ case, there are instances when the eddy does not span across the two sensors as illustrated in figure 10(b). Furthermore from the figure, it is evident that the portion of eddies that reach $y=\Delta s$ decreases linearly with increasing $\Delta s$ until $\Delta s=W_{i}$, from which point onwards the signals from the two sensors remain uncorrelated. Therefore, $\gamma_{L}^{2}=\max \left(1-\Delta s / \mathcal{W}_{i}, 0\right)$ for this idealised case, as illustrated in figure 10(d). In order to resolve the difference in $\gamma_{L}^{2}$ as a function of $\Delta s$ and $z$, here we propose that the linear $\gamma_{L}^{2}$ dependency with $\Delta s$ can be transformed to an equivalent step-like function observed for $z$ by preserving the area under the graph, in a similar manner to how an integral scale is inferred from $R_{u u}$. It should also be noted that the procedure is related to a 'coherence height' as defined by Jiménez et al. (2004). 
In the present case, however, the integration is carried out in the spanwise direction. Based on these arguments, we propose to extract $\mathcal{H}_{i}$ and $\mathcal{W}_{i}$ based on $z$ and $\Delta s$ locations where $\gamma_{L}^{2}=\Gamma$ (here $\Gamma$ corresponds to a threshold), leading to $\mathcal{H}_{i}=\left.z\right|_{\gamma_{L}^{2}=\Gamma}$ and $\mathcal{W}_{i}=\left.2 \Delta s\right|_{\gamma_{L}^{2}=\Gamma}$, respectively. For the streamwise extent since the coherence spectra are employed, $\mathcal{L}_{i}=\left.0.5 \lambda_{x}\right|_{\gamma_{L}^{2}=\Gamma}$, as negative $u$ fluctuations only remain coherent over half of the total wavelength, and because of association with the Fourier transform a positive $u$ fluctuation portion of the same size follows to form one full wavelength as indicated in figure 10(a) (blue and red regions denote negative and positive $u$, respectively). In the later section, we obtain the mean dimensions, $\mathcal{L}_{i} \times \mathcal{W}_{i} \times \mathcal{H}_{i}$, of the attached eddies that lead to a self-similar hierarchy in the logarithmic region, and show that they agree with previous studies that have examined the in-plane dimensions at a similar $R e$.

\subsection{Effects of Reynolds number}

Figure 11(a,(b)) shows the coherence spectra from the pipe flow at $R e_{\tau} \approx 10000$ and 40000 respectively. The grey scale and line contours again denote two transverse offset scenarios $\Delta s \approx 0$ and $\Delta s / R, \Delta s / \delta \approx 0.07$, as in figure 9 . Increasing $R e$ is accompanied by a larger scale separation between the viscous and inertial scales, and therefore also by an increase in the number of hierarchy levels required to model a wall-bounded flow. Indeed, this is reflected in an extended range of scales for which the $\gamma_{L}^{2}=0.1$ iso-contours (for the $\Delta s \approx 0$ scenario) follow the $\lambda_{x} \sim z$ scaling with increasing $R e$ for the pipe flows, as observed in figure 12. Also shown in figure 12 as vertical dotted lines are $\lambda_{x}^{+}=R_{x z} z_{\text {inertial }}^{+}$relations for each $R e$ case, where $z_{\text {inertial }}^{+}=2.6 \sqrt{R e_{\tau}}$ and $3.6 \sqrt{R e_{\tau}}$ for the pipe and boundary layer flows, respectively (Morrill-Winter et al. 2017; Wei et al. 2005). Here, $z_{\text {inertial }}$ corresponds to wall height where the mean viscous force first becomes sub-dominant in the mean momentum balance, and notably the $\gamma_{L}^{2}=0.1$ iso-contours in the near-wall region closely align with the $\lambda_{x}^{+}=R_{x z} z_{\text {inertial }}^{+}$relation for both the pipe and boundary layer flows. Note that the $z$-independent trend of $\gamma_{L}^{2}$ iso-contours observed in the experiment is not present in the coherence spectra from DNS (Baars et al. 2017). Hence, the experiments are unable to capture the wall-attached structures that reside in the region $z<z_{\text {inertial }}$. A potential cause for this is a small but finite spanwise misalignment that exists in the experiments between the skin friction and velocity sensors, which would lead to an attenuation of $\gamma_{L}^{2}$. A lower limit for the $\lambda_{x} \sim z$ scaling in the $\gamma_{L}^{2}=0.1$ iso-contours is indicative of the presence of different physical mechanisms of the wall-attached turbulence in the region close to the wall, which are not captured by the experiments. The current results suggest that these mechanisms are closely associated with an increased prominence of the viscous term in the mean momentum balance for the near-wall region (Morrill-Winter et al. 2017; Wei et al. 2005). It should be noted that the theory associated with the mean momentum balance shows that the scales of motions in the inertial domain are proportional to $z$, and thus the wall-distance scaling is an analytical result, rather than requiring assumptions as in $\mathrm{AEH}$.

Unlike the $\Delta s \approx 0$ case, an extension in the range of $\lambda_{x}$ values that remain coherent does not occur at $\Delta s / R, \Delta s / \delta \approx 0.07$ with $\operatorname{Re}$ (e.g. dashed lines in figures 11a,b). This behaviour can be related to the transverse offset remaining fixed in the outer scale for these datasets, and effectively acting as a low-pass filter based on the outer scale where scales smaller than the cutoff $\lambda_{x}$ do not remain coherent. In other words, the range of scales that are coherent is fixed between $O(0.1 R)$ and $O(R)$ for both cases, leading to coherent scales that are separated by a factor of $R / 0.07 R \sim O(10)$ regardless of $R e$. Furthermore, for the large-scale contributions, the iso-contours in figures 11(a) and (b) show good agreement, consistent with the AEH.

\subsection{Effects of transverse offset}

Figure 13 shows the effect of azimuthal offset $\Delta s$ for the $\gamma_{L}^{2}=0.1$ iso-contours from $R e_{\tau} \approx$ 40000 pipe flow. An azimuthal offset between the $u_{\tau}$ and $u$ sensors leads to a loss of common 

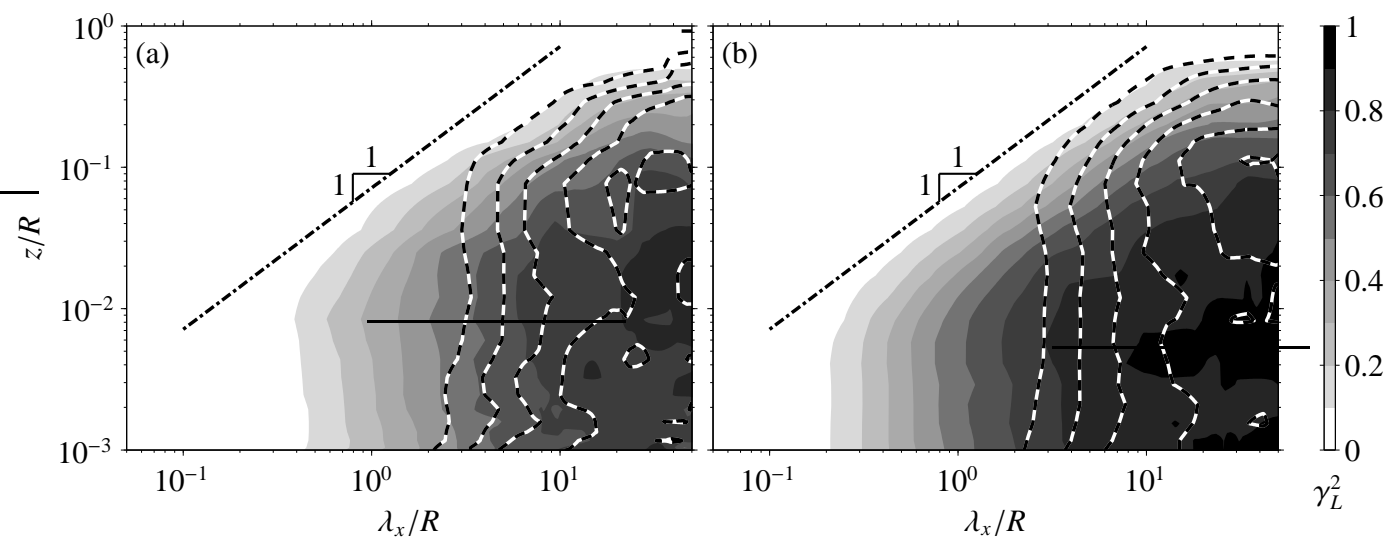

Figure 11: Value of $\gamma_{L}^{2}$ for a pipe flow at (a) $R e_{\tau} \approx 10000$ and (b) $R e_{\tau} \approx 40000$. The grey scale and line contours are as in figure 9.

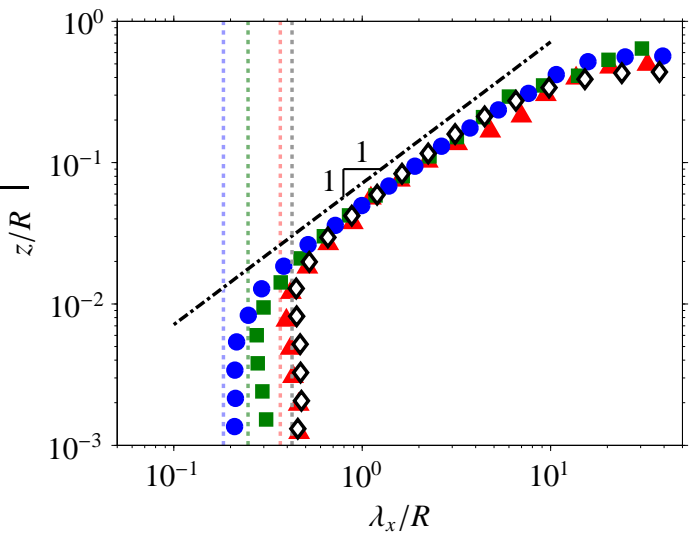

Figure 12: $\gamma_{L}^{2}=0.1$ contours between $u$ and $u_{\tau}$ signals at $\Delta s \approx 0$ for pipe and boundary layer flows. The symbols are as in figure 5, while the vertical dotted lines correspond to $\lambda_{x}^{+}=R_{x z} z_{\text {inertial }}^{+}$relations for each cases.

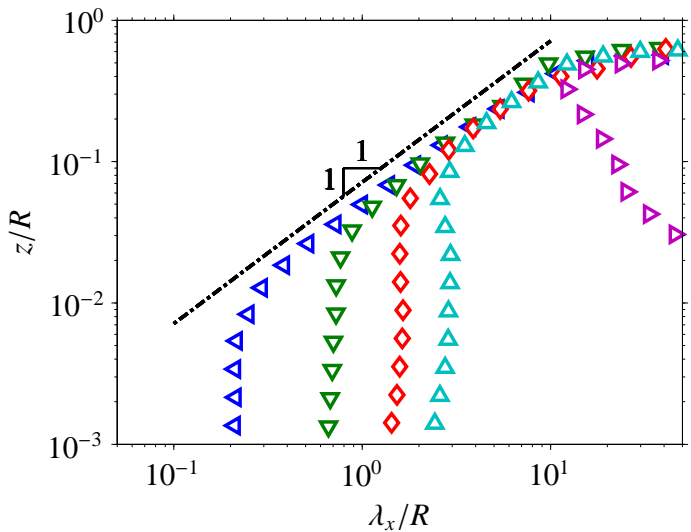

Figure 13: $\gamma_{L}^{2}=0.1$ contours between $u$ and $u_{\tau}$ signals at $R e_{\tau} \approx 40000$ as function of $\Delta s$; $\Delta s / R \approx 0(\triangleleft), 0.02(\nabla), 0.03(\diamond), 0.06(\triangle)$ and $0.13(\triangleright)$.

contributions encountered by the two sensors at the smaller scales since these scales do not span across the offset. Thus, a departure from the $\Delta s \approx 0$ contour occurs for any $\Delta s$ offsets larger than the width of the smallest attached eddy in the hierarchy, $\mathcal{W}_{1}$, with the departure occurring at a higher $\lambda_{x}$ when $\Delta s$ increases further.

To further assess the effect of transverse offset, figure 14 shows the coherence spectrum as a function of $\lambda_{x}$ and $\Delta s$. While figure 11(b) shows coherence spectra between a reference $u_{\tau}$ and $u$ as the $z$ offset is varied, in figure 14 the $\Delta s$ offset (see inset, symbols $\boldsymbol{\square}$ and $\bullet$ ) is varied instead. Similar to figure 11(b), two scenarios are considered where now the grey scale and line contour correspond to cases when the $u$ sensor is at $z / R \approx 0.01$ and 0.07 respectively (see inset, symbols $\square$ and $\square$ ). It should also be noted that figure 14(a,b) are the same, except for $\Delta s$ being shown in linear and logarithmic scale, respectively. The iso-contours at $z / R \approx 0.01$ and 0.07 agree well, at large $\lambda_{x}$ and $\Delta s$, while an increase in $z$ location where $u$ is acquired leads to a loss of coherence below a cutoff $\lambda_{x}$ that scales with the distance from the wall. Furthermore, unlike figure 11(b) the iso-contours do not obey a $\lambda_{x} \sim \Delta s$ (shown as dot-dashed line) scaling, 

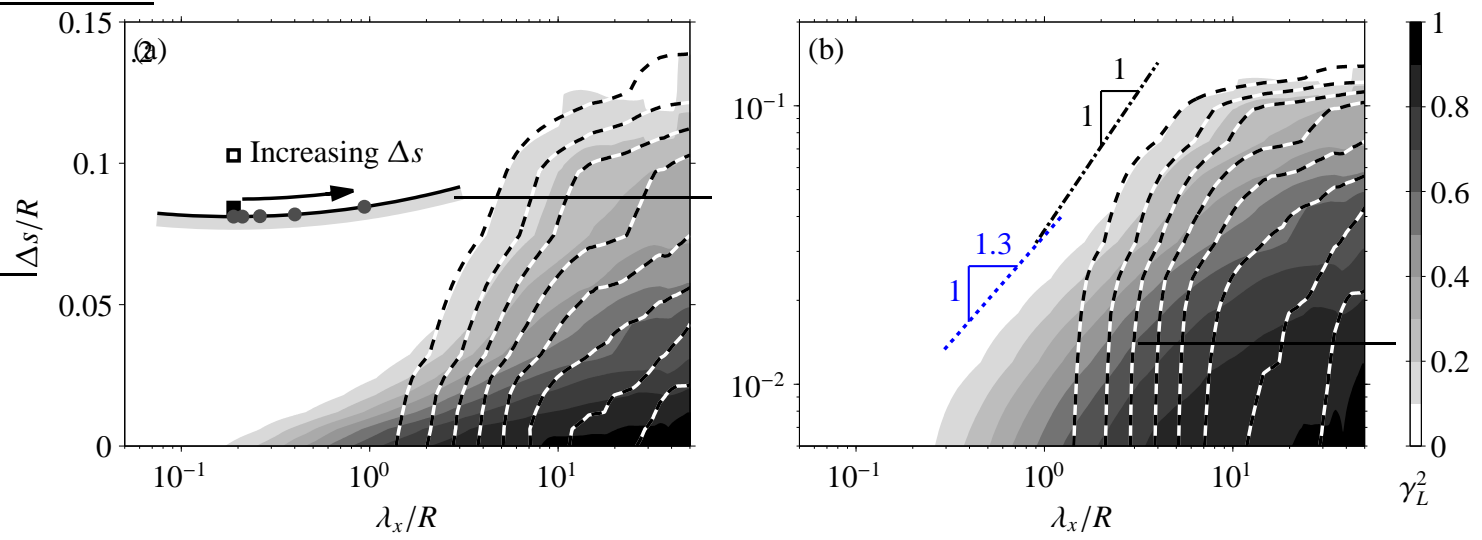

Figure 14: Value of $\gamma_{L}^{2}$, as a function of wavelength, $\lambda_{x}$, and hot-film sensor offset distances along the circumference, $\Delta s$, for the pipe flow at $R e_{\tau} \approx 40000$. The grey scale and line contours correspond to coherence when the hot-wire is at $z / R \approx 0.01(\square)$ and 0.07 ( $\square$ ) respectively, with the hot-film (๑) at an azimuthal offset of $\Delta s$. Both contours are at levels 0.1:0.1:0.9, while $\Delta s$ is shown in (a) linear and (b) logarithmic scale.

even at $R e_{\tau} \approx 40000$, and instead $\lambda_{x} \sim \Delta s^{1.3}$ behaviour is observed (shown as dotted line) for $\lambda_{x} / R \sim O(1)$ and $\Delta s / R \sim O(0.01)$. This is contrary to the AEH which predicts $\lambda_{x} \sim \Delta s$ behaviour owing to eddy self-similarity and the wall scaling in both the $y$ and $z$ directions. The failure of $\lambda_{x} \sim \lambda_{y}$ behaviour has also been noted by del Álamo et al. (2004) who examined the two-dimensional spectra of $u$ from DNS. This failure, however, could be related to low $R e$ effects, as Chandran et al. (2017) found evidence that the $\lambda_{x} \sim \lambda_{y}$ scaling may still emerge at the large scales at a higher $\operatorname{Re}\left(\operatorname{Re}_{\tau} \sim O\left(10^{6}\right)\right)$ leading to an emergence of $\lambda_{x} \sim \Delta s$ scaling for these scales. Hence, the current dataset at $R e_{\tau} \approx 40000$ may not be sufficiently high to see the $\lambda_{x} \sim \Delta s$ behaviour. Furthermore, similar to the $\lambda_{x} \sim \lambda_{y}$ scaling in the two-dimensional spectra, the $\lambda_{x} \sim \Delta s$ scaling of $\gamma_{L}^{2}$ contours are expected (at a sufficiently high $R e$ ) to first emerge at a $z$ location corresponding to the lower limit of the logarithmic region. This is because here the $u$ contributions from all hierarchy levels exist, while at a higher $z$ the contributions from hierarchy levels where $\mathcal{H}_{i}<z$ diminish. The dash-dotted line in figure 14 indicates $\lambda_{x}=R_{x y} \Delta s$, where $R_{x y}=28$ now is the predicted self-similar behaviour based on $\mathcal{H}_{i}=\mathcal{W}_{i}$ (i.e. $R_{x y}=2 R_{x z}$ since $\left.z\right|_{\gamma_{L}^{2}=\Gamma}=\left.2 \Delta s\right|_{\gamma_{L}^{2}=\Gamma}$, see figure 10). Furthermore, since $\lambda_{x}$ is expected to be twice as large as the region of streamwise coherence $\mathcal{L}_{i}$ (see figure 10a), this leads to an aspect ratio of $7: 1: 1$ for the self-similar eddy in the $x, y$ and $z$ directions, respectively. It has to be noted that Krug et al. (2019) found a clear dependence of this aspect ratio on the level of stratification, via analysis of atmospheric surface layer data. For our adiabatic wall case, the aspect ratio agrees well with the dimensions of attached eddies found in DNS, while the streamwise extent is slightly longer than that typically reported from DNS (del Álamo et al. 2006; Hwang 2015). However, the value obtained for the streamwise extent of the attached eddies is consistent with the observation of Chandran et al. (2017) that at a comparable $R e$ an energetic ridge in the two-dimensional $u$ spectra follow an aspect ratio of $7: 1$ in the $x$ and $y$ directions.

\subsection{Extending the model of Baars et al.}

Figure 15(a-d) shows a model for $\gamma_{L}^{2}$ based on results shown in $\S \S 4.1-4.3$ and following the AEH. In (a), $\gamma_{L}^{2}$ as a function of both $\lambda_{x}$ and $z$ is shown at a constant $\Delta s$, and hence the contours at the lower value of $\Delta s$ where $\Delta s \approx 0$ is equivalent to the model of Baars et al. (2017). In this model, the contribution to $\gamma_{L}^{2}$ from each hierarchy level remains uniform across $\lambda_{x}>R_{x z} \mathcal{H}_{i}$. The 
(a)

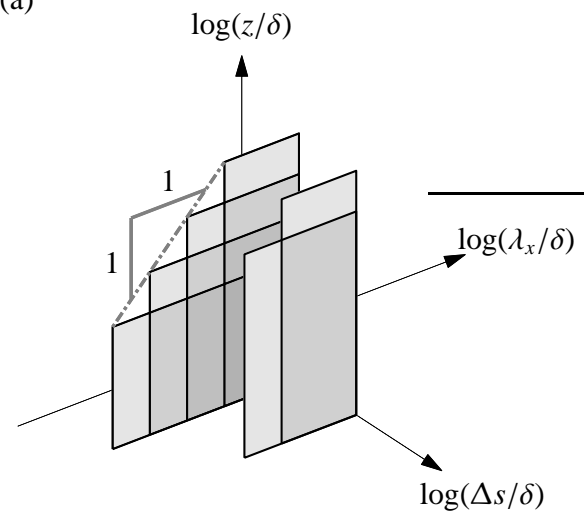

(c)

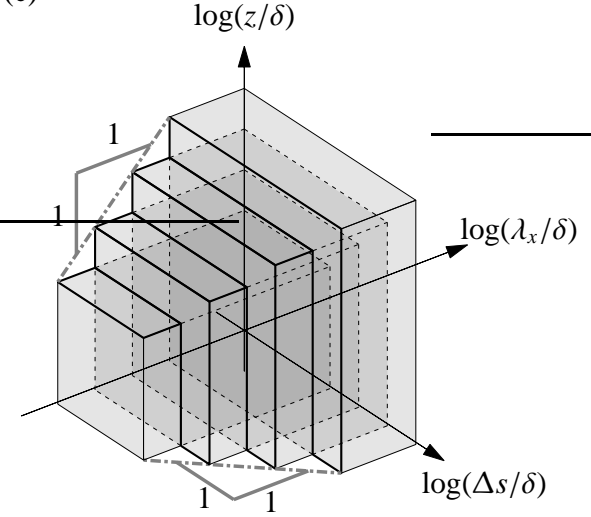

(b)

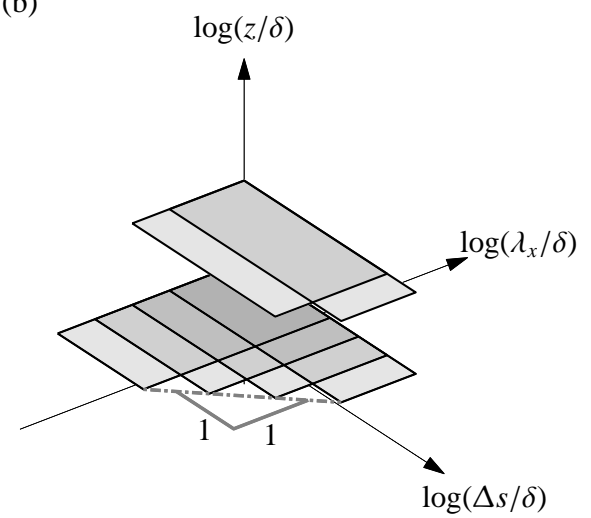

(d)

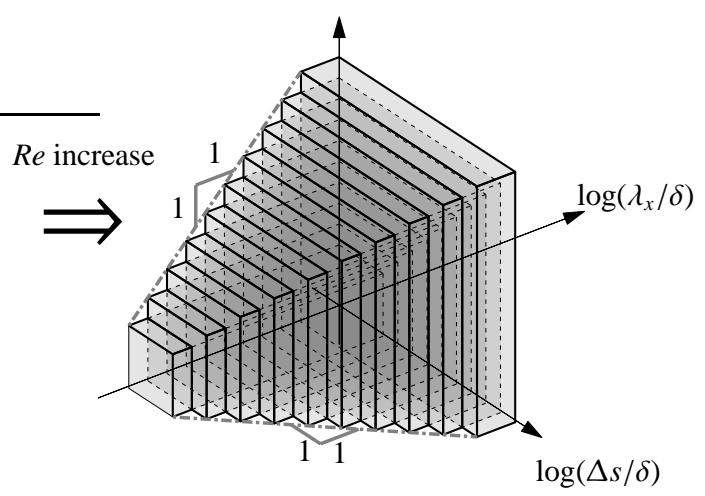

Figure 15: A model for $\gamma_{L}^{2}$ following the attached eddy hypothesis. (a-c) Correspond to the case with four hierarchy levels, with (a) and (b) showing $\gamma_{L}^{2}$ at a constant $\Delta s$ and $z$, while (c) illustrates the iso-contours of $\gamma_{L}^{2}$ as functions of $\lambda_{x}, \Delta s$ and $z$. Meanwhile, (d) shows the iso-contours for the case with ten hierarchy levels, which corresponds to a higher $R e$ scenario.

hierarchy scaling prescribed by the AEH, $\mathcal{H}_{i} \sim 2^{(i-1)} \mathcal{H}_{1}$, leads to a triangular region in the $\gamma_{L}^{2}$ iso-contours bounded by $R_{x z} \mathcal{H}_{1} \lesssim \lambda_{x} \lesssim \mathcal{R}_{x z} \mathcal{H}_{N_{H}}$. Here $N_{H}$ denotes the number of hierarchy levels used in the model, which is $R e$ dependent (e.g. $N_{H}=4$, for the examples shown in figures 8 and $15 \mathrm{a}-\mathrm{c}$ ). When $\Delta s>0.5 \mathcal{W}_{1}$, the lower bound of the triangular region increases, since an $i$ th hierarchy level with width $\mathcal{W}_{i}<2 \Delta s$ does not remain coherent across the two points, leading to $\gamma_{L}^{2} \approx 0$ at the smaller scales as shown in figure 15(a).

Figure 15(b) shows $\gamma_{L}^{2}$ as a function of $\Delta s$ and $\lambda_{x}$ at a constant $z$. Although the $\lambda_{x} \sim \Delta s$ scaling indicated in the figure did not materialise for the experimental data, evidence points towards its emergence at sufficiently high $R e$, when $R e_{\tau} \sim O\left(10^{6}\right)$ (Chandran et al. 2017). Thus in the asymptotic limit, the hierarchy of eddies are expected to be truly self-similar (i.e. the wall scaling holds in all three directions - $x, y$ and $z$ ), and therefore we expect a triangular region for the $\gamma_{L}^{2}$ contours in the $\lambda_{x}-\Delta s$ plane in an analogous manner to the $\lambda_{x}-z$ plane. Furthermore, the triangular region through this slice is now bounded by $R_{x z} \mathcal{W}_{1} \lesssim \lambda_{x} \lesssim R_{x z} \mathcal{W}_{N_{H}}$ (since $\mathcal{H}_{i} \sim \mathcal{W}_{i}$ ) when $z<\mathcal{H}_{1}$, since contributions to $\gamma_{L}^{2}$ from all hierarchy levels are captured at those locations (see figure 15b at a lower $z$ location). Beyond this wall height, the lower bound of the triangular region increases due to loss of coherence from the smaller members of the hierarchy.

Combining the results from figure 15(a,(b)), figure 15(c) depicts the full three-dimensional 
picture of $\gamma_{L}^{2}$ contours as a function of $\lambda_{x}, \Delta s$ and $z$. Here, the triangular regions observed on constant $\Delta s$ and $z$ planes now correspond to the face of a skewed pyramid, as eddies that do not meet the $\mathcal{W}_{i}>2 \Delta s$ and $\mathcal{H}_{i}>z$ criteria do not remain coherent over the two points used to calculate $\gamma_{L}^{2}$. At a higher $R e$, the number of hierarchy levels required in the model increases, leading to a more extended pyramidal region for the $\gamma_{L}^{2}$ iso-contours as shown in figure $15(\mathrm{~d})$.

\section{Summary and conclusions}

Experiments in the large-scale pipe and boundary layer facilities are conducted to examine wall-attached structures at high Reynolds number. The use of large-scale facilities enables access to high Reynolds number flows while retaining resolvable small-scale features using conventional measurement techniques. For the pipe experiment, an array of 51 azimuthally spaced skin friction sensors are simultaneously sampled in concert with a traversing velocity sensor. The boundary layer experiment features an array of 10 spanwise-spaced skin friction sensors, which are also simultaneously acquired with a velocity sensor. The velocity measurements, in conjunction with a reference skin friction signal at the wall, are used for a coherence analysis in the spectral domain (Baars et al. 2016). Furthermore, the array of skin friction sensors enables examination of the linear coherence spectrum, $\gamma_{L}^{2}$, as a function of the streamwise wavelength $\left(\lambda_{x}\right)$, azimuthal/spanwise offset $(\Delta s)$ and wall-normal offset $(z)$.

At a comparable Reynolds number, the pipe and boundary layer $\gamma_{L}^{2}$ exhibit a similar dependence on $\lambda_{x}, \Delta s$ and $z$. In general, a reduction in $\gamma_{L}^{2}$ is observed as the two points are moved apart due to loss of contributions from the common structure spanning across the two points. When normalised by the respective outer length scales, the differences between the pipe and boundary layer flows is evident for $\gamma_{L}^{2}$ in the wake region. However, in the logarithmic region (where the effect of the boundary conditions is minimal), good agreement in $\gamma_{L}^{2}$ is observed between the pipe and boundary layer; namely, the iso-contours of $\gamma_{L}^{2}$ exhibit a $\lambda_{x} \sim z$ behaviour in both flows (see figure 9). This is consistent with the attached eddy hypothesis (Townsend 1976), which assumes the $\lambda_{x} \sim z$ scaling due to the existence of a hierarchy of self-similar eddies that scale with distance from the wall. Moreover, the region where the present empirical observations indicate self-similar behaviour correspond tp where the mean momentum equation formally admits a self-similar invariant form, and simultaneously where the mean and variance profiles of the streamwise velocity exhibit logarithmic dependence.

A loss in coherence at an increased azimuthal/spanwise separation between the velocity and skin friction sensors leads to a reduction in the range of scales that follow the $\lambda_{x} \sim z$ scaling. Thus, a hierarchy of self-similar eddies is expected to manifest a pyramidal region for the $\gamma_{L}^{2}$ iso-contours in $\left(\lambda_{x}, \Delta s, z\right)$ space as shown in figure 15 . Based on experimental observations, we presume these self-similar eddies to follow an aspect ratio of $7: 1: 1$ in the streamwise, spanwise and wall-normal directions, respectively.

\section{Acknowledgement}

The authors gratefully acknowledge the financial support from the Australian Research Council and the European High-Performance Infrastructures in Turbulence (EuHIT) program.

\section{REFERENCES}

Adrian, R. J., Meinhart, C. D. \& Tomkins, C. D. 2000 Vortex organization in the outer region of the turbulent boundary layer. J. Fluid Mech. 422, 1-54.

Ahn, J., Lee, J. H., Lee, J., KAng, J. \& Sung, H. J. 2015 Direct numerical simulation of a $30 R$ long turbulent pipe flow at $R e_{\tau}=3008$. Phys. Fluids 27 (6), 065110. 
Alfredsson, P. H., Johansson, A. V., Haritonidis, J. H. \& Eckelmann, H. 1988 The fluctuating wall-shear stress and the velocity field in the viscous sublayer. Phys. Fluids 31 (5), 1026-1033.

BaArs, W. J., Hutchins, N. \& Marusic, I. 2016 Spectral stochastic estimation of high-Reynolds-number wall-bounded turbulence for a refined inner-outer interaction model. Phys. Rev. Fluids 1 (5), 054406.

BaARs, W. J., Hutchins, N. \& Marusic, I. 2017 Self-similarity of wall-attached turbulence in boundary layers. J. Fluid Mech. 823, R2.

Baidya, R., Philip, J., Hutchins, N., Monty, J. P. \& Marusic, I. 2017 Distance-from-the-wall scaling of turbulent motions in wall-bounded flows. Phys. Fluids 29 (2), 020712.

Bellani, G. \& Talamelli, A. 2016 The final design of the long pipe in CICLoPE. In Progress in turbulence $V I$, pp. 205-209. Springer.

Bendat, J. S. \& Piersol, A. G. 2010 Random data: analysis and measurement procedures, 4th edn. John Wiley \& Sons.

Chandran, D., Baidya, R., Monty, J. P. \& Marusic, I. 2017 Two-dimensional energy spectra in highReynolds-number turbulent boundary layers. J. Fluid Mech. 826, R1.

Chauhan, K., Philip, J., de Silva, C. M., Hutchins, N. \& Marusic, I. 2014 The turbulent/non-turbulent interface and entrainment in a boundary layer. J. Fluid Mech. 742, 119-151.

Chung, D., Marusic, I., Monty, J. P., Vallikivi, M. \& Smits, A. J. 2015 On the universality of inertial energy in the log layer of turbulent boundary layer and pipe flows. Exp. Fluids 56 (7), 1-10.

Davidson, P. A. \& Krogstad, P. - $\AA$. 2008 On the deficiency of even-order structure functions as inertialrange diagnostics. J. Fluid Mech. 602, 287-302.

Del Álamo, J. C. \& Jiménez, J. 2009 Estimation of turbulent convection velocities and corrections to Taylor's approximation. J. Fluid Mech. 640, 5-26.

del Álamo, J. C., Jiménez, J., Zandonade, P. \& Moser, R. D. 2004 Scaling of the energy spectra of turbulent channels. J. Fluid Mech. 500, 135-144.

del Álamo, J. C., Jiménez, J., Zandonade, P. \& Moser, R. D. 2006 Self-similar vortex clusters in the turbulent logarithmic region. J. Fluid Mech. 561, 329-358.

Hellström, L. H., Marusic, I. \& Smits, A. J. 2016 Self-similarity of the large-scale motions in turbulent pipe flow. J. Fluid Mech. 792, R1.

Hutchins, N. \& Marusic, I. $2007 a$ Evidence of very long meandering features in the logarithmic region of turbulent boundary layers. J. Fluid Mech. 579, 1-28.

Hutchins, N. \& Marusic, I. $2007 b$ Large-scale influences in near-wall turbulence. Philos. Trans. R. Soc. London, Ser. A 365, 647-664.

Hutchins, N., Monty, J. P., Ganapathisubramani, B., Ng, H. C. H. \& Marusic, I. 2011 Three-dimensional conditional structure of a high-Reynolds-number turbulent boundary layer. J. Fluid Mech. 673, 255285.

Hutchins, N., Nickels, T. B., Marusic, I. \& Chong, M. S. 2009 Hot-wire spatial resolution issues in wallbounded turbulence. J. Fluid Mech. 635, 103-136.

Hwang, Y. 2015 Statistical structure of self-sustaining attached eddies in turbulent channel flow. J. Fluid Mech. 767, 254-289.

JiMÉnEZ, J. 2011 Cascades in wall-bounded turbulence. Annu. Rev. Fluid Mech. 44 (1), 27.

JimÉnez, J., Del ÁlAmo, J. C. \& Flores, O. 2004 The large-scale dynamics of near-wall turbulence. J. Fluid Mech. 505, 179-199.

Kim, K. C. \& Adrian, R. J. 1999 Very large-scale motion in the outer layer. Phys. Fluids 11 (2), 417-422.

Krug, D., BaArs, W. J., Hutchins, N. \& Marusic, I. 2019 Vertical coherence of turbulence in the atmospheric surface layer: connecting the hypotheses of Townsend and Davenport. Boundary Layer Meteorol. .

Kwon, Y. 2016 The quiesecent core of turbulent channel and pipe flows. PhD thesis, University of Melbourne.

Kwon, Y. S., Hutchins, N. \& Monty, J. P. 2016 On the use of the Reynolds decomposition in the intermittent region of turbulent boundary layers. J. Fluid Mech. 794, 5-16.

Ligrani, P. M. \& Bradshaw, P. 1987 Spatial resolution and measurments of turbulence in the viscous sublayer using subminature hot-wire probes. Exp. Fluids 5, 407-417.

Marusic, I., McKeon, B. J., Monkewitz, P. A., Nagib, H. M., Smits, A. J. \& Sreenivasan, K. R. 2010 Wallbounded turbulent flows at high Reynolds numbers: recent advances and key issues. Phys. Fluids 22 (6), 065103.

Marusic, I. \& Monty, J. P. 2019 Attached eddy model of wall turbulence. Annu. Rev. Fluid Mech. 51, 49-74. 
Marusic, I., Monty, J. P., Hultmark, M. \& Smits, A. J. 2013 On the logarithmic region in wall turbulence. J. Fluid Mech. 716, 716.

Mathis, R., Hutchins, N. \& Marusic, I. 2009 Large-scale amplitude modulation of the small-scale structures in turbulent boundary layers. J. Fluid Mech. 628, 311-337.

MonTy, J. P. 2005 Developments in smooth wall turbulent duct flows. PhD thesis, The University of Melbourne.

Monty, J. P., Hutchins, N., Ng, H. C. H., Marusic, I. \& Chong, M. S. 2009 A comparison of turbulent pipe, channel and boundary layer flows. J. Fluid Mech. 632, 431-442.

Monty, J. P., Stewart, J. A., Williams, R. C. \& Chong, M. S. 2007 Large-scale features in turbulent pipe and channel flows. J. Fluid Mech. 589, 147-156.

Morrill-Winter, C., Philip, J. \& Klewicki, J. 2017 An invariant representation of mean inertia: theoretical basis for a log law in turbulent boundary layers. J. Fluid Mech. 813, 594-617.

Nickels, T. B., Marusic, I., Hafez, S. \& Chong, M. S. 2005 Evidence of the $k_{1}^{-1}$ law in a high-Reynoldsnumber turbulent boundary layer. Phys. Rev. Lett. 95 (7), 074501.

Örlü, R., Fiorini, T., Segalini, A., Bellani, G., Talamelli, A. \& Alfredsson, P. H. 2017 Reynolds stress scaling in pipe flow turbulence - first results from CICLoPE. Philos. Trans. R. Soc. London, Ser. A 375 (2089), 20160187.

Perry, А. E. \& Chong, M. S. 1982 On the mechanism of wall turbulence. J. Fluid Mech. 119, 173-217.

Perry, A. E., Marusic, I. \& Jones, M. B. 2002 On the streamwise evolution of turbulent boundary layers in arbitrary pressure gradients. J. Fluid Mech. 461, 61-91.

Piomelli, U., Balint, J.-L. \& Wallace, J. M. 1989 On the validity of Taylor's hypothesis for wall-bounded flows. Phys. Fluids 1 (3), 609-611.

Robinson, S. K. 1991 Coherent motions in the turbulent boundary layer. Annu. Rev. Fluid Mech. 23 (1), 601-639.

Sillero, J. A., Jiménez, J. \& Moser, R. D. 2014 Two-point statistics for turbulent boundary layers and channels at Reynolds numbers up to $\delta^{+} \approx 2000$. Phys. Fluids 26 (10), 105109.

de Silva, C. M., Gnanamanickam, E. P., Atkinson, C., Buchmann, N. A., Hutchins, N., Soria, J. \& Marusic, I. 2014 High spatial range velocity measurements in a high Reynolds number turbulent boundary layer. Phys. Fluids 26 (2), 025117.

de Silva, C. M., Marusic, I., Woodcock, J. D. \& Meneveau, C. 2015 Scaling of second-and higher-order structure functions in turbulent boundary layers. J. Fluid Mech. 769, 654-686.

Smits, A. J., McKeon, B. J. \& Marusic, I. 2011 High-Reynolds number wall turbulence. Annu. Rev. Fluid Mech. 43, 353-375.

Talamelli, A., Persiani, F., Fransson, J. H. M., Alfredsson, P. H., Johansson, A. V., Nagib, H. M., Rüedi, J., Sreenivasan, K. R. \& Monkewitz, P. A. 2009 CICLoPE - a response to the need for high Reynolds number experiments. Fluid Dyn. Res. 41 (2), 021407.

Taylor, G. I. 1938 The spectrum of turbulence. Proc. R. Soc. London, Ser. A 164 (919), 476-490.

Tennekes, H. \& Lumley, J. L. 1972 A first course in turbulence. MIT Press.

Townsend, A. A. 1976 The structure of turbulent shear flow, 2nd edn. Cambridge Univ Press.

Wei, T., Fife, P., Klewicki, J. \& McMurtry, P. 2005 Properties of the mean momentum balance in turbulent boundary layer, pipe and channel flows. J. Fluid Mech. 522, 303-327.

Willert, C. E., Soria, J., Stanislas, M., Klinner, J., Amili, O., Eisfelder, M., Cuvier, C., Bellani, G., Fiorini, T. \& Talamelli, A. 2017 Near-wall statistics of a turbulent pipe flow at shear reynolds numbers up to 40 000. J. Fluid Mech. 826, R5.

Yang, X. I. A., Baidya, R., Johnson, P., Marusic, I. \& Meneveau, C. 2017 Structure function tensor scaling in the logarithmic region derived from the attached eddy model of wall-bounded turbulent flows. Phys. Rev. Fluids 2 (6), 064602.

Zagarola, M. V. \& Smits, A. J. 1998 Mean-flow scaling of turbulent pipe flow. J. Fluid Mech. 373, 33-79. 